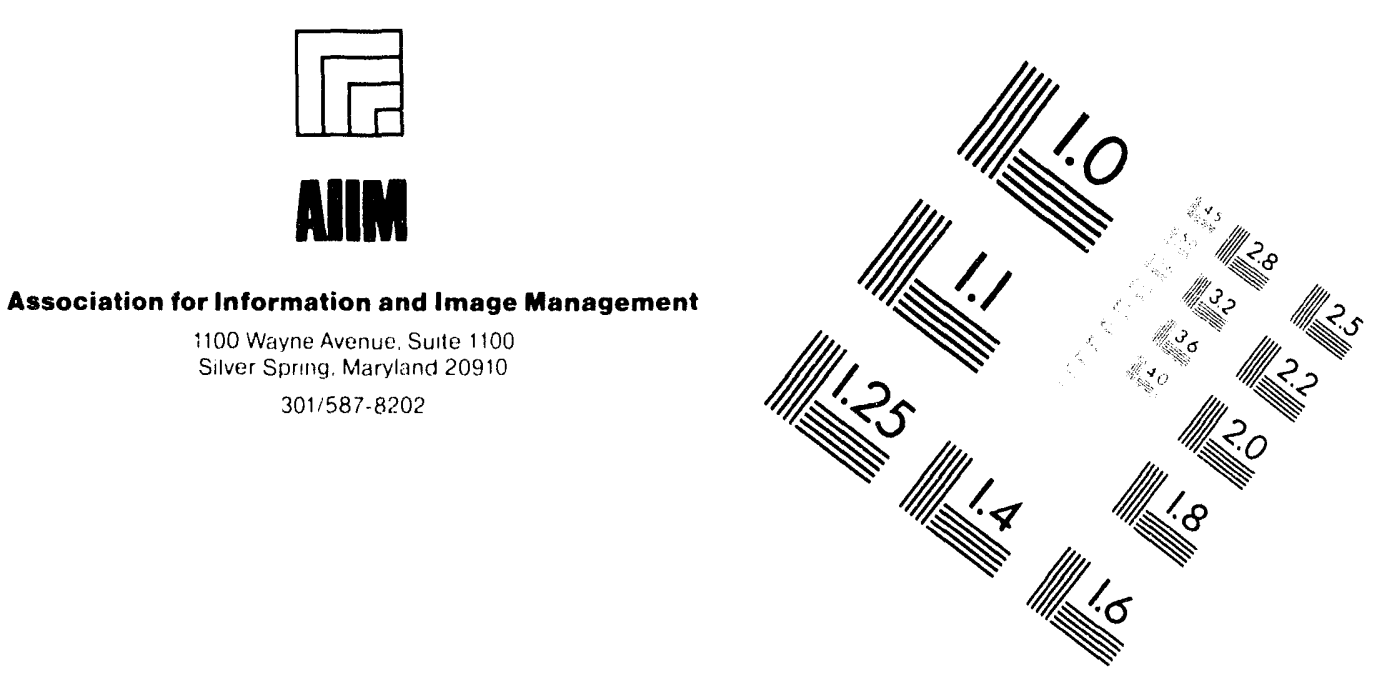

Centimeter

||

Inches
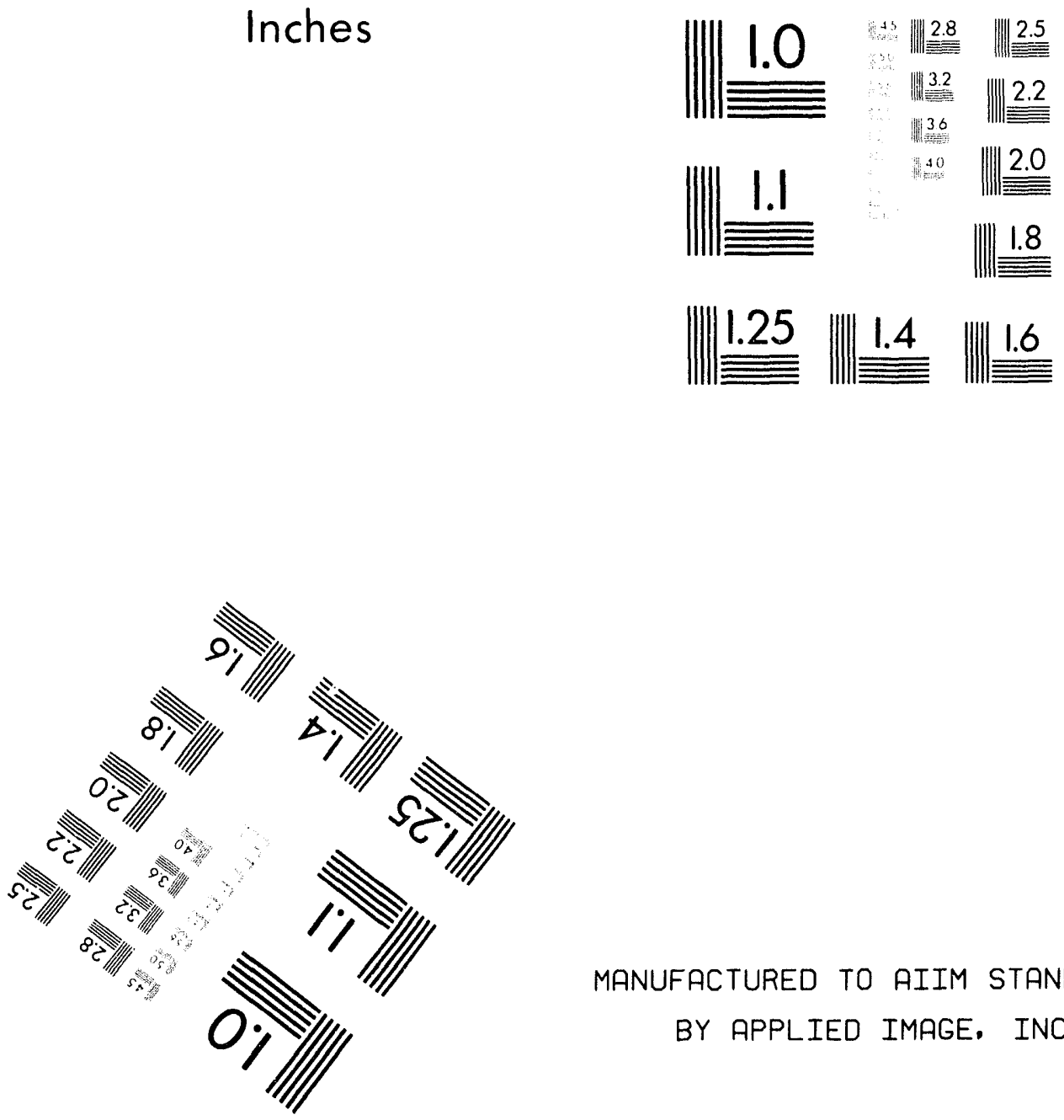

MANUFACTURED TO AIIM STANDARDS

BY APPLIED IMAGE, INC.

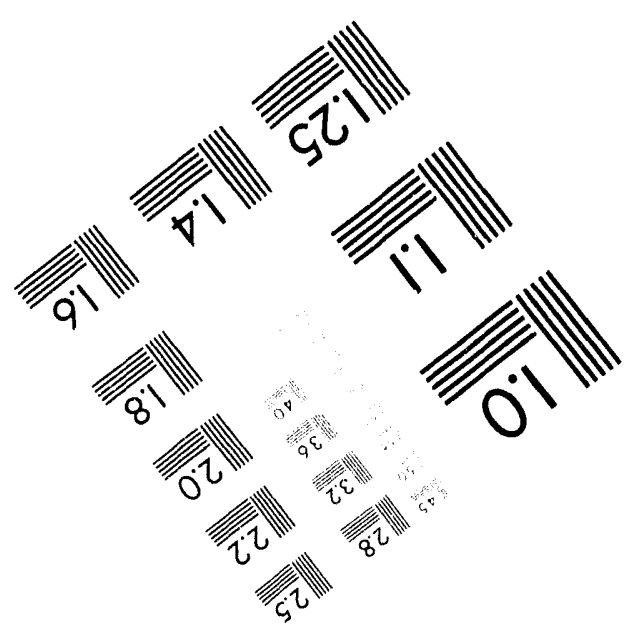



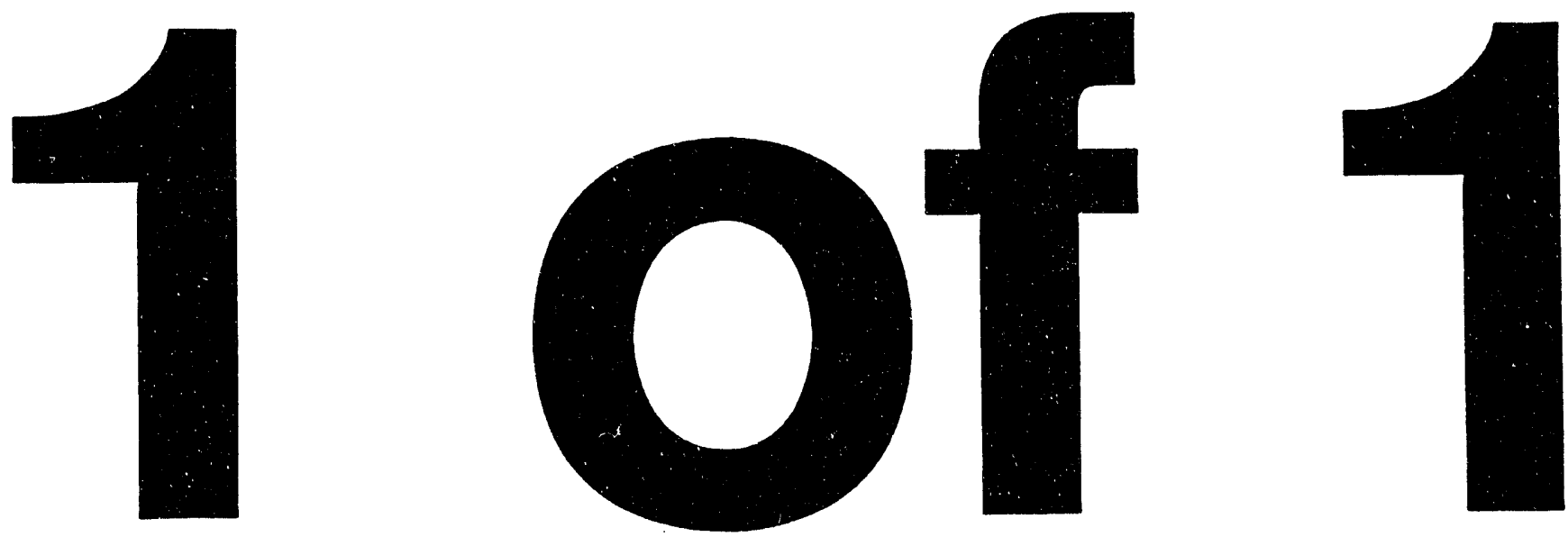


\section{IDAHO NATIONAL ENGINEERING LABORATORY ENVIRONMENTAL RESTORATION PROGRAM SCHEDULE CONTINGENCY EVALUATION REPORT}

Published September 1993

\section{DISCLAIMER}

This report was prepared as an account of work sponsored by an agency of the United States Government. Neither the United States Government nor any agency thereof, nor any of their employees, makes any warranty, express or implied, or assumes any legal liability or responsibility for the accuracy, completeness, or usefulness of any information, apparatus, product, or process disclosed, or represents that its use would not infringe privately owned rights. Reference herein to any specific commercial product, process, or service by trade name, trademark, manufacturer, or otherwise does not necessarily constitute or imply its endorsement, recommendation, or favoring by the United States Guvernment or any agency thereof. The views and opinions of authors expressed herein do not necessarily state or reflect those of the United States Government or any agency thereof.

Prepared for the

U.S. Department of Energy

DOE Idaho Operations Office

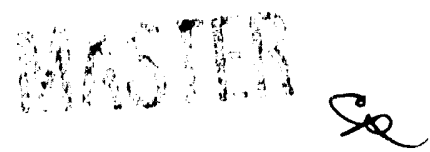




\begin{abstract}
This report represents the schedule contingency evaluation done on the FY-93 Major System Acquisition (MSA) Baseline for the Idaho National Engineering Laboratory's (INEL) Environmental Restoration Program (ERP). A Schedule Contingency Evaluation Team (SCET) was established to evaluate schedule contingency on the MSA Baseline for the INEL ERP associated with completing work within milestones established in the baseline. Baseline schedules had been established considering enforceable deadlines contained in the Federal Facilities Agreement/Consent Order (FFA/CO), the agreement signed in 1992, by the State of Idaho, Department of Health \& Welfare, the U. S. Environmental Protection Agency, Region 10, and the U. S. Department of Energy, Idaho Operations Office. The evaluation was based upon the application of standard schedule risk management techniques to the specific problems of the INEL ERP. The schedule contingency evaluation was designed to provided early visibility for potential schedule delays impacting enforceable deadlines. The focus of the analysis was on the duration of time needed to accomplish all required activities to achieve completion of the milestones in the baseline corresponding to the enforceable deadlines. Additionally, the analysis was designed to identify control of high-probability, high-impact schedule risk factors.
\end{abstract}




\title{
CONTENTS
}

\author{
ABSTRACT \\ EXECUTIVE SUMMARY \\ OVERVIEW \\ DEVELOPMENT OF CONFIDENCE RATINGS \\ ANALYSIS PLAN \& DATA REQUIREMENTS \\ DATA COLLECTION METHODGLOGY \\ TREND ANALYSIS \\ APPENDIX A - INEL SCHEDULE RISK EVALUATION TEAM \\ APPENDIX B - INSTRUCTIONS \& DATA FORMS \\ APPENDIX C - SCHEDULE WITH RISK ADDED \\ APPENDIX D - DATA TABLES
}




\section{IDAHO NATIONAL ENGINEERING LABORATORY ENVIRONMENTAL RESTORATION SCHEDULE CONTINGENCY EVALUATION}

\section{EXECUTIVE SUMMARY}

The schedule contingency evaluation for the Idaho National Engineering Laboratory (INEL) Environmental Restoration Program (ERP) was developed based upon standard risk evaluation techniques. Risk was quantified in terms of time and defined in terms of the FY-93 Major Systems Acquisition (MSA) Baseline milestones corresponding to the enforceable deadlines established in the Federal Facilities Agreement/Consent Order (FFA/CO). Table 1 lists the FFA/CO enforceable deadlines that were determined to be "at risk".

Table 1. FFA/CO Enforceable Deadlines at Risk

\begin{tabular}{|l|l|l|l|}
\hline WAG 4 & Landfills RI/FS & OU 4-12 & $\begin{array}{l}\text { Draft ROD Submitted to EPA/IDHW for } \\
\text { Review }\end{array}$ \\
\hline WAG 4 & $\begin{array}{l}\text { Comprehensive } \\
\text { RI/FS }\end{array}$ & OU 4-13 & $\begin{array}{l}\text { Draft ROD Submitted to EPA/IDHW for } \\
\text { Review }\end{array}$ \\
\hline WAG 5 & $\begin{array}{l}\text { Comprehensive } \\
\text { RI/FS }\end{array}$ & OU 5-12 & $\begin{array}{l}\text { Draft RI/FS SOW Submitted to EPA/IDHW } \\
\text { for Review }\end{array}$ \\
\hline WAG 7 & Pad A RI/FS & OU 7-12 & $\begin{array}{l}\text { Draft ROD Submitted to EPA/IDHW for } \\
\text { Review }\end{array}$ \\
\hline WAG 7 & $\begin{array}{l}\text { Comprehensive } \\
\text { RI/FS }\end{array}$ & OU 7-14 & $\begin{array}{l}\text { Draft ROD Submitted to EPA/IDHW for } \\
\text { Review }\end{array}$ \\
\hline WAG 10 & $\begin{array}{l}\text { Comprehensive } \\
\text { RI/FS }\end{array}$ & OU 10-04 & $\begin{array}{l}\text { Draft RI/FS Submitted io EPA/IDHW for } \\
\text { Review }\end{array}$ \\
\hline WAG 10 & $\begin{array}{l}\text { Comprehensive } \\
\text { RI/FS }\end{array}$ & OU i0-04 & $\begin{array}{l}\text { Draft ROD Submitted to EPA/IDHW for } \\
\text { Review }\end{array}$ \\
\hline
\end{tabular}

Schedule contingency factors were determined in a bottoms up approach at the Cost Account level where operations managers determined their confidence in meeting the established MSA Baseline schedule. A two step process was used to obtain confidence levels, additional schedule time estimates based upon identified contingency, and causality. Each of the steps is identified below:

1. The Department of Energy (DOE) Idaho Operations Office (ID) Waste Area Group (WAG) managers in conjunction with the appropriate cost account managers and 
members of the SRET selected a limited number of MSA Baseline milestones that provided a clear picture of the cost account activities on the critical path for FFA/CO enforceable deadlines. The activities between selected milestones were "Hammocked" together to identify a subproject for this evaluation. The total duration of the hammock in the MSA Baseline was considered the time allowed to complete all tasks and meet the end-point selected milestone corresponding to the FFA/CO enforceable deadline.

2. Confidence ratings of Ahead, On, Behind and Late (A, O, B, L) were assigned to each hammock by the appropriate cost account manager depending on his/her confidence in being able to accomplish all tasks required to meet the selected end-point milestone in the time specified in the FY-93 Baseline. These confidence ratings were not associated with any set value and were purely subjective. The level of resolution for meeting established baseline schedules was + or - one week. Scope and budget were to be considered constants as defined in the MSA Baseline. In all cases where Ahead, Behind or Late were selected by the individuals responsible for schedule planning and compliance, the cost account managers, they were required to quantify the contingency associated with their confidence rating and explain the contingency and quantification. If they were not confident in the schedule, they determined the time difference between the current schedule and a schedule that they would have confidence in meeting.

The schedule contingency was added to the hammocked durations and a "contingencyadded" schedule recomputed for each operating unit (OI), WAG and the ERP. These contingency-added schedules were compared to the FFA/CO enforceable deadlines and possible problems identified. "At risk" deadlines were defined as any enforceable deadline with less than 14 days float between the deadline date and the corresponding planning milestone date. In each instance where the manager did not have confidence in meeting the established schedule, the causes of his/her concerns were determined and the controlling individuals, agencies or organizations identified. During detailed evaluations, certain logic errors were observed in the MSA Baseline schedule. These errors were corrected and the corrections communicated to those involved in the FY-94 Cost Account Plan development to ensure that the next set of planning data incorporated appropriate logic.

\section{OVERVIEW}

For all Environmental Restoration Programs (ERP), contingency is an integral part of the integrated Baseline for scope, schedule, and cost. At the Idaho National Engineering Laboratory (INEL), the contingency associated with implementing the ER Major Systems Acquisition (MSA) Baseline have been successfully addressed with regards to cost estimates. However, the contingency in meeting the schedule established in the MSA Baseline, which was designed to 
meet requirements of the Federal Facilities Agreement/Consent Order (FFA/CO) had not been evaluated in any detail. A Schedule Contingency Evaluation Team (SCET) was established to assess schedule contingency and evaluate schedule risk for the INEL ERP associated with enforceable deadlines contained in the FFA/CO. The team was sponsored by the U.S. Department of Energy Idaho Operations Office (DOE-ID), and composed of representatives from Chem-Nuclear Geotech Inc., EG\& G Idaho Inc., Westinghouse Idaho Nuclear Company (WINCO), and MK-Ferguson of Idaho Company Environmental Restoration Program (MK-FIC-ERP). Team members are listed in Appendix A. This report identifies schedule contingency associated with individual operating units and rolls-up that contingency to Waste Area Group (WAG) and total ER Program schedules and FFA/CO enforceable deadlines.

Schedule risk identified in this report is defined as the additional time required beyond the established schedule to perform the scope of work defined in the baseline based upon the uncertainties associated with those activities. Schedule contingency was evaluated at a macro level where schedule durations were viewed for a combination of activities between important milestones including the currently scheduled float between activities within the hammock. The focus of the analysis was on the duration of time needed to accomplish all required activities to achieve completion of the milestones in the baseline corresponding to the enforceable deadlines.

Schedule risk was evaluated during May 1993, for all of the environmental restoration activities except for those expected to result in a No Further Action decision, decontamination and decommissioning activities, state and tribal grants, technical support contractor activities, program management activities, and other direct-funded DOE-ID activities.

\section{DEVELOPMENT OF CONFIDENCE RATINGS}

In order to maintain the macro level perspective, it was decided that subjective evaluations of confidence in the established schedule, taking into account the known and unknown contingency, would be used. Four levels of confidence were selected that represented the possible schedule performance for the selected subprojects. The confidence ratings used were: (A) confident in completion ahead of schedule, $(0)$ confident in completion on schedule, (B) probably complete behind schedule, and $(\mathrm{L})$ confident that project would be late. Probability was not quantified but was considered accounted for in the cost account managers confidence ratings. The managers responsible for project completion were selected as the source of the confidence rating and other required data.

\section{ANALYSIS PLAN \& DATA REQUIREMENTS}

The analysis plan was designed to evaluate the schedules for selected milestones in the INEL ERP MSA Baseline that directly supported the FFA/CO enforceable deadlines and the 
activities in the schedule that were on the critical path for those milestones. The focus of the analysis was on the duration of time needed to accomplish all required activities to achieve completion of the milestones supporting the enforceable deadlines. FFA/CO deadlines would be identified as "at contingency" if the schedule had less than two week of float between the planning milestone and the deadline. Trend analysis was needed to evaluate any program-wide schedule contingency. All contingency associated threats to the established schedule needed to be identified, the nature of those threats identified and the extent of the impact on the schedule evaluated. Three data points were needed for each subproject to fulfill these evaluation requirements. These data points were: schedule confidence ratings, schedule differentials due to risk, and risk associated causality.

In order to limit the number of activities and milestones to a manageable number for analysis, the DOE WAG managers selected the most important milestones for their cost account level projects. Then the SCET developed subprojects from the MSA Baseline schedule by hammocking together the activities for the selected milestones. Subproject durations were determined by taking the difference between the scheduled start for the first activity in a hammock and the associated completion for the milestone identifying the completion of the hammocked activities.

The confidence of the operational cost account managers in completing the activities within a hammock, and therefore meeting the milestones, was the indicator for possible problems associated with schedule contingency. The impact of the contingency on the schedule required quantification. This was accomplished by having the operational cost account managers identify the causes of their lack of confidence in the established schedules for the identified subprojects and what schedule changes would be necessary for them to be confident in a new schedule. The level of resolution for schedule confidence and schedule contingency associated differentials used in the analysis was plus or minus one week.

The analysis plan required that integrated logic networks for precedence schedules be developed from the MSA Baseline for the hammocked subprojects. The contingency adjusted durations would then be applied and a new set of "contingency added" logic networks produced. By comparing these to networks, the schedule contingency would be visible and could be easily compared to the FFA/CO enforceable deadlines. Schedule contingency for the program was evaluated by comparing the program schedule in the MSA Baseline, reflected in the integrated schedule for the subprojects, with the integrated program schedule adjusted for the additional time required to mitigate identified schedule contingency. This time differential was added (or subtracted in cases of being ahead of schedule) to the duration and a new integrated schedule calculated with contingency. The "contingency-added" schedule was then overlaid with the FFA/CO enforceable deadlines.

The data was to be compiled and verified by the SCET by comparing confidence ratings with estimated differentials in weeks. Logic diagrams were prepared for the selected subprojects 
at the WAG and Program level, with and without contingency. Color-coded Gantt charts for the operating units within each WAG were prepared to display the impact of contingency on the MSA Baseline schedule. The FFA/CO deadlines were overlaid on the Gantt charts and the enforceable deadlines "at risk" identified.

Trend analysis was accomplished by evaluating the identified causes listed for the confidence ratings of behind or late for similar subprojects.

\section{DATA COLLECTION METHODOLOGY}

A single data collection effort was designed to minimize the time required to collect the three data points for each hammocked subproject from the operational cost account managers. Data forms and instructions were developed by the SCET (see Appendix B) and distributed with a one week suspense. The forms were partially filled out by the SCET and identified the OU, the end-point milestone, the duration of the hammock/subproject, and the precedence between this hammock and other hammocks in the logic schedule. The cost account managers were instructed to provide contingency quantification and causality for all subprojects where their confidence rating was other than "on schedule". The data forms were collected and the data compiled into the integrated schedules for each cost account. The cost account data was at the operating unit $(O U)$ level which was then rolled-up into WAG and program level schedules and compared to the FFA/CO deadlines.

\section{RESULTS}

Seven FFA/CO deadlines in six Operating Units were identified as "at risk" in this evaluation, but only two are "at risk" due to identified schedule uncertainty. The other five deadlines were at risk due to logic errors in the schedule in the MSA Baseline for FY-93. All the other FFA/CO deadlines had sufficient float in their FY-93 schedules to accommodate schedule uncertainties and identified logic errors.

During the development of the integrated logic networks for the OUs, WAGs and the ERP from the FY-93 MSA Baseline, logic planning errors were discovered where interfaces between activities were missing. Therefore, delays in one activity did not directly pass on to subsequent activities in the same projects and subsequent activities within the same WAG or between WAGs. Corrected logic networks were constructed and new schedules computed. The impact of these correction are indicated in blue on the schedules in Appendix C. This has resulted in three enforceable deadlines "at risk". The other two deadlines "at risk" are due to current planning with minimum float.

The MSA FY-93 Baseline planning dates for all the selected milestones used in this 
evaluation are contained in Appendix D. The table also contains a column for corrected dates for milestones that were affected by planning errors. The third column is for milestone "contingency-added" dates and a fourth column for applicable enforceable FFA/CO deadlines. By comparing the dates for the milestones, the impact of contingency can be determined as well as the float remaining.

Operating unit schedules were developed from the integrated logic networks, both uncorrected and corrected, and with contingency factors added. These charts are in Appendix C.

The schedules in Appendix $\mathrm{C}$ show a single bar for all the subprojects within an OU, the applicable milestones (triangles) and FFA/CO deadlines (vertical bars). The schedules and milestones are color-coded for the FY-93 MSA Baseline (green), a corrected version of the baseline resolving the planning errors (blue), and contingency-add impacts (purple). FFA/CO deadlines are indicated by a black vertical bar and interfaces between the WAGs are in magenta. All FFA/CO deadlines at risk are indicated by a diamond in the color indicating the cause of the problem (original baseline planning, planning errors, or risk).

Trend analysis was unable to identify any trends in the causes of schedule contingency due to the small number of subprojects where schedule confidence was low. Individual samples were evaluated to attempt to determine who controlled the causes or processes the resulted in the identified schedule contingencies. Control of the identified significant schedule contingency factors could not be determined. The inability to achieve consensus and unexpected complexity of tasks were examples of the schedule contingency factors for which control cannot be determined. Delays in document reviews and approvals were caused by a variety of factors and again control was not determined.

The mitigation of schedule contingency's impact on the MSA Baseline schedule can be accomplished by planning for the identified schedule contingency while process improvement investigations are conducted to identify and eliminate the causes of schedule contingency.

\section{CONCLUSIONS}

The INEL ERP schedule is not "at risk". The timely identification of schedule contingency factors and existing planning errors has permitted corrective actions to be implemented through the FY-94 Cost Account Plan development process and development of the FY-94 MSA Baseline schedule.

A follow-on schedule contingency evaluation of the FY-94 MSA Baseline is recommended to verify effectiveness of the implemented corrective actions and identify any new contingency factors that could impact the schedule. A higher level of resolution is also 
recommended for the analysis and data collection to identify the causes of schedule contingency and facilitate the development of process improvements to eliminate them. The time period for this evaluation is recommended to be the second quaiter of FY-94, with the report due no latter that the end of the third quarter of FY-94. 
APPENDIX A

INEL Schedule Contingency Evaluation Team 


\title{
INEL Schedule Contingency Evaluation Team
}

\author{
U. S. Department of Energy, Idaho Field Office \\ K. R. Hastings
}

\section{RUST Geotech Inc.}
R. A. Matthews
J. E. Bruce
B. K. Seggerty

EG\&G Idaho, Inc. Environmental Restoration
M. G. Koll
J. L. Butler
D. D. Shafer

Westinghouse Idaho Nuclear Company Environmental Restoration

L. L. Hutterman

MK-Ferguson of Idaho Company Environmental Restoration Program

D. A. Groetzinger

D. A. Fisher 
APPENDIX B

DATA Forms \& Instructions 


\section{Guidance for Completion of Schedule Contingency Data Sheets}

1. No additional assumptions are permitted other than those used to complete the current Baseline. Assume no change in funding or scope.

2. The quality of the product, if there is a deliverable associated with the milestone, must be assured. The normal quality assurance process cannot be cut out to meet schedules.

3. Schedule risks are to be considered in the confidence rating. Assume that preceding milestones over which you have no control are being met.

4. This effort will only address the most significant milestones. CAP-level critical path milestones, milestones that are likely to become critical path due to risk, significant milestones with less than two weeks of float and milestones with interfaces should be considered for inclusion. Generally, no more than 5 milestones within a single cost account would be evaluated. 
Column 1: Provide the work package number in which the milestone is contained.

Column 2: Provide the unique logic identification number for the milestone.

Column 3: Waste Area Group and Operable Unit in which the milestone resides.

Column 4: Milestone description as it appears in the work package. No modifications should be made to the text.

Column 5: Total duration of the hammock from the beginning task to the milestone. Hammocks should include all tasks preceding the accomplishment of the milestone and may include less significant milestones within the hammock.

Column 6: Baseline date on which the milestone is scheduled to be finished.

Column 7: Work package number for the milestone which directly precedes the one being evaluated. If none, so state.

Column 8: Unique identification number for the milestone which directly precedes the one being evaluated.

Column 9: Work package number for the milestone which directly follows the one being evaluated. If none, so state.

Column 10: Unique identification number for the milestone which directly follows the one being evaluated.

Column11: Classify your milestone completion estimate in one of the following ways:
A - will be completed ahead of schedule
$O$ - will be completed on schedule ( $+/-1$ week)
$B$ - behind schedule with moderate assurance of schedule risk estimate
L - behind schedule with low assurance of schedule risk estimate


Column 12: Estimate, in weeks, deviation from the baseline schedule.

Column 13: Provide clear, concise explanations for the deviation from the baseline date. 
Cost Account Title

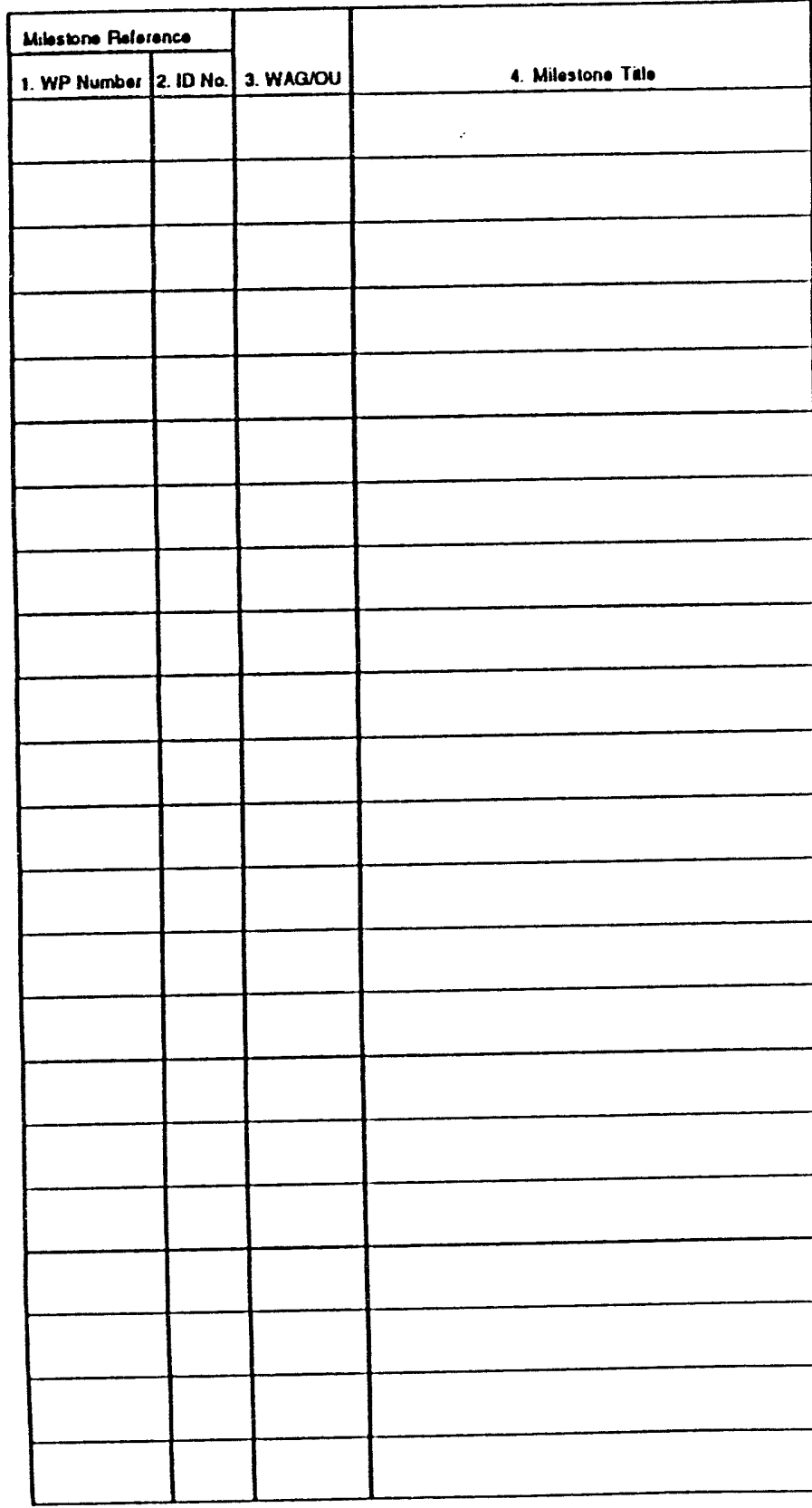

Cost Account Number

\begin{tabular}{|c|c|c|c|c|c|c|}
\hline 5. Hammock & 8. Bassatino & 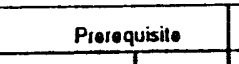 & Succossor & \begin{tabular}{|l|l} 
11. Complation \\
\end{tabular} & 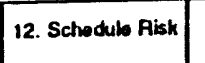 & 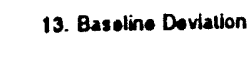 \\
\hline & fuish Date & & 2. WP Numboof $\mid 10.10 \mathrm{No}$ a & Chassilicallon & (Wootal & \\
\hline & & & & & & \\
\hline & & & & & & \\
\hline & & & & & & \\
\hline & & & & & & \\
\hline & & & & & & \\
\hline & & & & & & \\
\hline & & & & & & \\
\hline & & & & & & \\
\hline & & & & & & \\
\hline & & & & & & \\
\hline & & & & & & \\
\hline & & & & & & \\
\hline & & & & & & \\
\hline & & & & & & \\
\hline & & & & & & \\
\hline & & & & & & \\
\hline & & & & & & \\
\hline & & & & & & \\
\hline & & & & & & \\
\hline & & & & & & \\
\hline & & & & & & \\
\hline & & & & & & \\
\hline
\end{tabular}




\section{Schedule Contingency Worksheet \\ Waste Area Group 1}

Page 1 ol 2

\begin{tabular}{|c|c|c|c|c|c|c|c|c|c|c|c|c|}
\hline \multirow{3}{*}{\multicolumn{2}{|c|}{ Cost Account Tillo }} & \multirow{2}{*}{\multicolumn{4}{|c|}{ WAG 1 Track 2 OUs }} & \multirow{2}{*}{\multicolumn{4}{|c|}{ Cost Account Number 3XNA21000 }} & \multirow{4}{*}{$\begin{array}{l}\text { 11. Completion } \\
\text { Classitication }\end{array}$} & \multirow{4}{*}{$\begin{array}{l}\text { 12. Schodule } \\
\text { Pisk (Wooks) }\end{array}$} & \multirow{4}{*}{$\begin{array}{l}\text { 13. Baseline Dovistion } \\
\text { Basis }\end{array}$} \\
\hline & & & & & & & & & & & & \\
\hline & & \multirow[b]{2}{*}{ 3. Wharou } & \multirow[b]{2}{*}{ 4. Milosione Tille } & \multirow{2}{*}{$\begin{array}{c}\text { 5. Harmoock } \\
\text { Durstion (Wooks) }\end{array}$} & \multirow{2}{*}{$\begin{array}{l}\text { 6. Besoline } \\
\text { Finish Date }\end{array}$} & \multicolumn{2}{|c|}{ Pioroqulsite } & \multicolumn{2}{|l|}{ Suocesson } & & & \\
\hline 1. Wes Numbor & $2.10 \times 5$. & & & & & \multirow{2}{*}{ 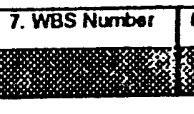 } & \multirow{2}{*}{ 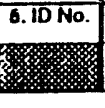 } & \multirow{2}{*}{\begin{tabular}{c|} 
O. WOS Nuntoer \\
1.1 .1 .1 .4 .1 .1 \\
\end{tabular}} & \multirow{2}{*}{\begin{tabular}{|c|}
$10.10 \mathrm{No}$ \\
37 \\
\end{tabular}} & & & \\
\hline T.1.1.1.2.1.4 & 94 & $1-\infty$ & 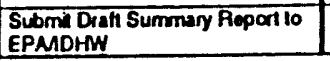 & 65.4 & $2 / 3 / 106$ & & & & & $\overline{0}$ & 0 & NA \\
\hline \multicolumn{2}{|c|}{ Cost Account TIlle } & \multicolumn{4}{|c|}{ WAG 1 Comprehensive Al/FS } & \multicolumn{4}{|c|}{ Cost Account Numbor $\quad 3 \times N A 32000$} & & & \\
\hline \multicolumn{2}{|c|}{ Milestom Palecence } & \multirow[b]{2}{*}{ 3. WAONOU } & \multirow[b]{2}{*}{ 4. Mibstone Tile } & \multirow{2}{*}{$\begin{array}{c}\text { 5. Hammock } \\
\text { Durabion (Wooks) }\end{array}$} & \multirow{2}{*}{$\begin{array}{l}\text { C. Basolino } \\
\text { Finish Dale }\end{array}$} & \multicolumn{2}{|l|}{ Proroquisise } & \multicolumn{2}{|l|}{ Suocess $\alpha$} & & & 13. Baseline Deviation \\
\hline 1. WBS Nunbor & $2.10 \mathrm{No}$ & & & & & 7. WES Nurribot & 8.10 No. & O. WES Nunbor & $10.10 \mathrm{No}$ & Clesgitication & Pisk (Wooks) & Basis \\
\hline 1.1.1.1.4.1.1 & 37 & $1-10$ & $\begin{array}{l}\text { Drant SOW SLbmitod to } \\
\text { EPMDHW }\end{array}$ & 18.3 & 81805 & $\begin{array}{l}1.1 .1 .2 .1 .4 \\
1.1 .1 .131 .1\end{array}$ & 24 & 1.1.1.1.4.1.1 & 30 & O & $\overline{0}$ & NA \\
\hline 1.1.1.1.4.1.1 & 30 & $1-10$ & $\begin{array}{l}\text { Submi Drath Work Plan to } \\
\text { EPMDrW }\end{array}$ & 77.1 & $10 / 23105$ & $\frac{1.1 .1 .1 .1 .1 .1}{10}$ & 37 & $\overline{1.1 .1 .1 .4 .1 .2}$ & 24 & $\overline{0}$ & $\overline{0}$ & TWh \\
\hline 1.1.1.1.4.1.2 & 24 & 1.10 & 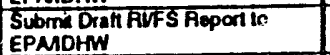 & 01,2 & 817797 & $\begin{array}{l}1.1 .1 .1 .4 .1 .1 \\
1.1 .1 .1 .6 .1 .1\end{array}$ & $\begin{array}{l}30 \\
04\end{array}$ & 1.1 .1 .1 .4 .1 .3 & $\begin{array}{l}10 \\
39\end{array}$ & O & 0 & WA \\
\hline 1.1.1.1.4.1.3 & 10 & $1-10$ & Submin Drah hOD 10 EPNMOHW & 43.0 & $\sqrt{30 / 98}$ & 1.1.1.1.1.1.2 & $\frac{04}{24}$ & $\frac{1.1 .10 .1 .1 .1 .2}{1.1 .1 .1 .4 .1 .3}$ & $\frac{39}{23}$ & 0 & $\overline{0}$ & $\sqrt{A}$ \\
\hline 1.1.1.1.4.1.3 & 23 & 1.10 & Final hoo Approved & 17.0 & 83 yod & 1.1.1.1.4.1.3 & 10 & 1.1 .1 .2 .010 & 450 & o & 0 & NA \\
\hline Cost Account & Tille & TAN Grour & ndwater RI/FS & & & Cost Acc & count Nu & 3XNA3 & 1000 & & & \\
\hline Mtestome Pale & rences & & & 5. Harmmock & 6. Baseline & Proroquisite & & Suocesso & & 11. Complotion & 12. Sctredulo & 13. Baseline Deviation \\
\hline 1. WBS Numbor & 2.10 No. & 3. WAGNOU & 4. Mllertone Tite & Duration (Weeks) & Finish Date & 7. Was Number & $8.10 \mathrm{No}$ & O. WeS Number & 10.10 No. & Classitication & Fisk (Wooks) & Basiz \\
\hline I.1.1.1.3.1.1 & 24 & 1078 & $\begin{array}{l}\text { Swbmi Drah AVFS Roportio } \\
\text { EPMDHM }\end{array}$ & 16.1 & $7 / 2803$ & & & $\begin{array}{l}1.1 .1 .1 .3 .1 .2 \\
1.1 .1 .1 .1 .1\end{array}$ & $\begin{array}{l}16 \\
37\end{array}$ & $\bar{\theta}$ & $\overline{2}$ & $\begin{array}{l}\text { Theroased comploxiny d report is } \\
\text { causing a complation dolaty. }\end{array}$ \\
\hline 1.1.1.1.3.1.2 & 46 & $1-078$ & Submi Drath AOO to EPMIOHW & 40.0 & $5 / 17194$ & 1.1.1.1.3.1.1 & 24 & $\frac{0.1 .1 .1 .3 .1 .2}{2}$ & 35 & o & 0 & NA \\
\hline 1.1.1.1.3.1.2 & 35 & 1.076 & Final hOO Approved & 18.1 & $10 / 394$ & 1.1.1.1.3.1.2 & 18 & 1.1 .12 .078 & 450 & o & D & WA \\
\hline Cost Account & TIlle & Inlerim Ac & lion Planning TSF-07 & & & Cosl Acc & count $\mathrm{Nu}$ & 3XNA4 & 1000 & & & \\
\hline Milestono Pole & rence & & & 5. Hammock & C. Baseline & Pioroqulsin & & Suocessor & & 11. Complotion & 12. Schodulo & 13. Basoline Doviation \\
\hline 1. Wós Number & $2.16 \mathrm{No}$ & 3. WaGrov & 4. Mibstono Tillo & Duration (Weeks) & Finish Dale & 7. WBS Numbor & 8.10 No. & O. WOS Nuntol & $10.10 \mathrm{No}$ & Classilication & Pisk (Wooks) & Basis \\
\hline 1.1.1.1.0.1.1 & क0 & 1.00 & Submi Drath SOW lo EPM:DHW & 5.0 & 4/NOS & & & 1.1.1.1.6.1.1 & 84 & 0 & 0 & NA \\
\hline T.1.1.1.0.1.1 & 84 & 1.08 & Subme Drah hOD to EPNDHW & $44 . \overline{0}$ & $2 / 23 / 80$ & 1.1.1.1.6.1.1 & 10 & $\begin{array}{l}\text { T.1.1.1.6.1.1 } \\
\text { 1.1.1.1.1.2 }\end{array}$ & $\begin{array}{l}15 \\
24\end{array}$ & o & 0 & WA \\
\hline 1.1.1.1.0.1.1 & 45 & $1-\infty$ & Final AOD Approved & 17.3 & $627 / 96$ & 1.1.1.1.8.1.1 & 84 & 1.1 .1 .006 & 450 & 0 & 0 & TWA \\
\hline Cost Account & Tille & OU 1-7B T & TAN Groundwater RI/FS & & & Cost Acc & count $\mathrm{Nu}$ & 1.1.1.2. & $7 \mathrm{~B}$ & & & \\
\hline Mitestono Rele & dence & & & 5. Hammock & 6. Basoline & Prosequisite & & Successo & & 11. Complotion & 12. Sctrodule & 12. Basoline Devizion \\
\hline 1. Wes Number & $2.10 \mathrm{Ko}$ & 3. HAGJOU & 4. Mibstone Talle & Duration (Weeks) & Fintsh Da10 & 7. WES Number & D. $10 \mathrm{No}$ & D. WBS Nuntbot & 10.10 No. & Classitication & Risk (Wooks) & Basis \\
\hline 1.1.1.2.078 & 450 & $1-078$ & $\begin{array}{l}\text { Subma bran SOWFA Work PLan } \\
\text { TOEPANOHW }\end{array}$ & 13,0 & 102404 & 1.1 .1 .1 .3 .1 .2 & 35 & $\begin{array}{l}1.1 .12 .078 \\
1.1 .12 .078\end{array}$ & $\begin{array}{l}196 \\
213\end{array}$ & o & 0 & NA \\
\hline$\overline{1.1 .1 .2 .078}$ & 106 & 1078 & $\begin{array}{l}\text { Submi Dran Dosign } 10 \\
\text { EPMDrW D }\end{array}$ & 18.0 & $3 / 13 / 95$ & $\begin{array}{l}1.1 .1 .2 .078 \\
1.1 .12078\end{array}$ & $\begin{array}{l}150 \\
213\end{array}$ & $\begin{array}{l}1.1 .12 .078 \\
1.1 .12 .078 \\
\end{array}$ & $\begin{array}{l}617 \\
917\end{array}$ & o & 0 & NA \\
\hline 7.1 .1 .078 & $\overline{213}$ & $1-078$ & $\begin{array}{l}\text { Submin Drant au Work Ptan to } \\
\text { EPMOHFW }\end{array}$ & 18.0 & 31395 & 1.1.1.2.078 & 450 & $\begin{array}{l}1.112 .078 \\
1.112 .078 \\
1.112 .078\end{array}$ & $\begin{array}{l}617 \\
917 \\
198\end{array}$ & 0 & 0 & $\overline{\text { NA }}$ \\
\hline 1.1.1.2.078 & 617 & i-078 & 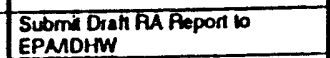 & 87.1 & $12 / 8 / 90$ & $\begin{array}{l}T 1.1 .1 .2 .078 \\
1.1 .12078\end{array}$ & $\begin{array}{l}108 \\
213 \\
\end{array}$ & & & o & $\overline{0}$ & $\overline{\mathbf{N A}}$ \\
\hline 1.1.1.2.078 & 017 & $1-078$ & $\begin{array}{l}\text { EPMuUin Drall OsM Ropon } 10 \\
\text { SPMDHW }\end{array}$ & 80.4 & V/1097 & $\begin{array}{l}1.1 .12 .078 \\
1.1 .12 .078\end{array}$ & $\begin{array}{l}106 \\
213\end{array}$ & & & 0 & 0 & WA \\
\hline
\end{tabular}




\section{Schedule Contingency Worksheet Waste Area Group 1}

Page 2 of 2

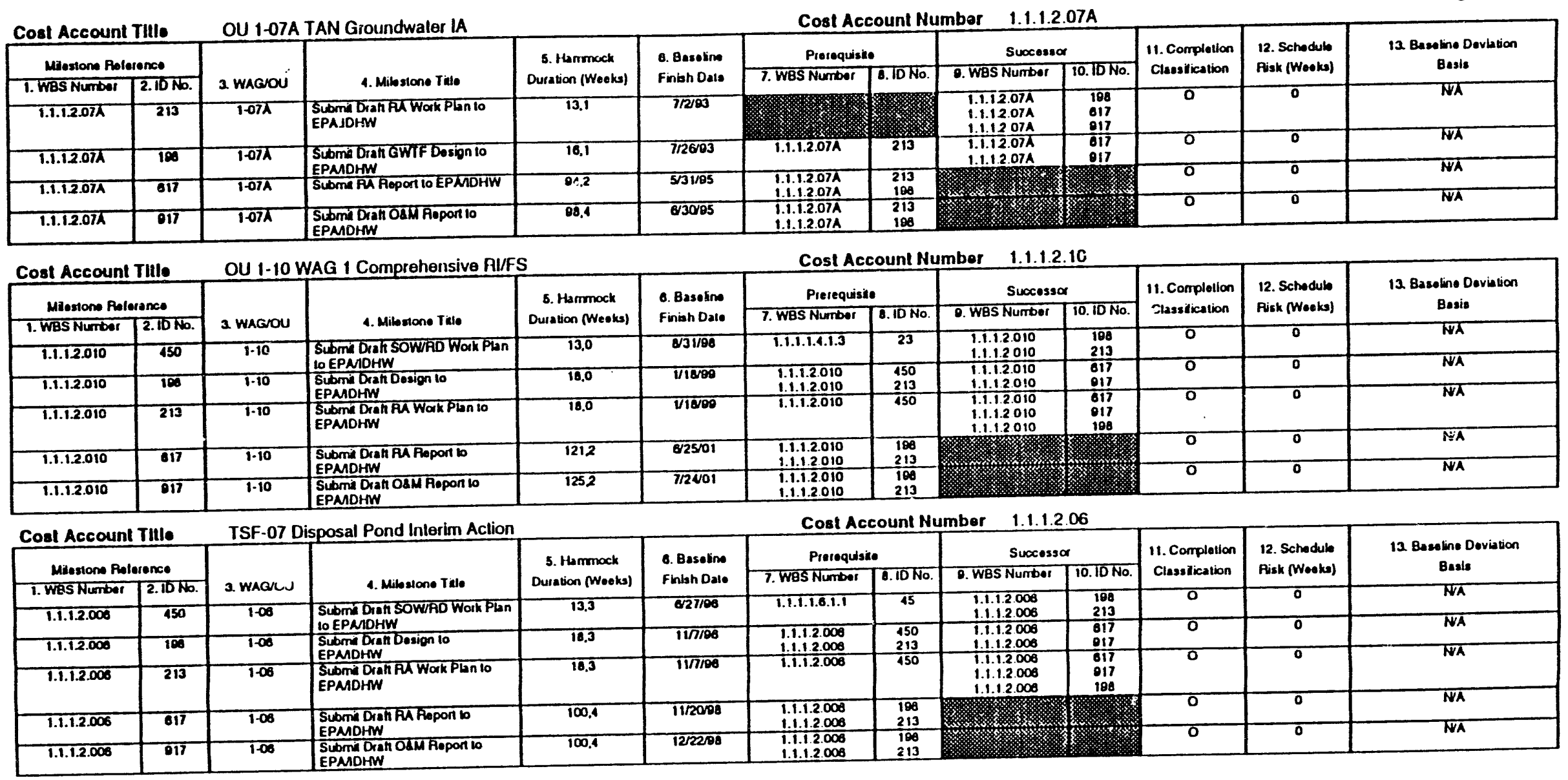




\section{Schedule Contingency Worksheet}

Waste Area Group 2

\begin{tabular}{|c|c|c|}
\hline \multicolumn{2}{|c|}{ Minstiong folorence } & \multirow[b]{2}{*}{ 3. WAGOU } \\
\hline 1. Was Number & $2.10 \mathrm{Na}$ & \\
\hline$\overline{1.12 .2 .000}$ & 450 & $2-\infty$ \\
\hline 1.1 .2 .2 .000 & 10 & $2-\infty$ \\
\hline 1.1 .2 .2 .000 & 213 & $2-00$ \\
\hline 1.1 .2 .2 .000 & 617 & $2-00$ \\
\hline 1.1.2.2.000 & 817 & 200 \\
\hline
\end{tabular}

Cost Account Number

1.1.2.2.9

\begin{tabular}{|c|c|}
\hline 4. Milostone Tíle & $\begin{array}{l}\text { 5. Hammock } \\
\text { Duation (Wooke) }\end{array}$ \\
\hline 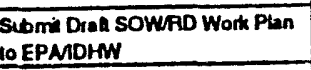 & 13.0 \\
\hline Submh Drat Dogign 10 EPMDTHW & 10.0 \\
\hline $\begin{array}{l}\text { Submi Dinh Pu Woak Plan bo } \\
\text { EPMDHwW }\end{array}$ & 10.0 \\
\hline $\begin{array}{l}\text { Subrii Drath PM Fopont to } \\
\text { EPMDHW }\end{array}$ & 78.0 \\
\hline $\begin{array}{l}\text { Submi Diath OSM Fopon to } \\
\text { EPMDHW }\end{array}$ & 82,1 \\
\hline
\end{tabular}

\begin{tabular}{|c|}
\hline $\begin{array}{l}\text { O. Baseline } \\
\text { Finish Dade }\end{array}$ \\
\cline { 2 - 2 } \\
\hline $7 / 28 / 94$ \\
\hline $12 / 12 / 94$ \\
\hline $12 / 12 / 94$ \\
\hline $7 / 10 / 80$ \\
\hline $8 / 8 / 88$ \\
\hline
\end{tabular}

Prorequalite

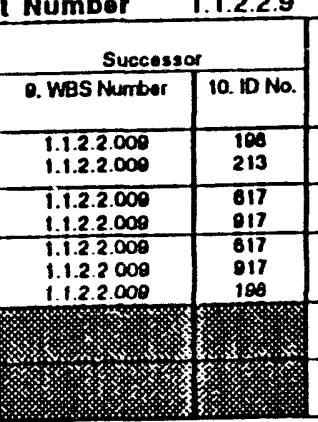

\begin{tabular}{|c|c|c|}
\hline $\begin{array}{l}\text { 11. Complation } \\
\text { Clessilicallon }\end{array}$ & $\begin{array}{l}\text { 12. Schodule } \\
\text { Plisk } \\
\text { (Woeks) }\end{array}$ & $\begin{array}{l}\text { 13. Basoline Deviation } \\
\text { Besis }\end{array}$ \\
\hline TNA & 0 & $\begin{array}{l}\text { Thiorim Aaton wil nol bo } \\
\text { pertormod. }\end{array}$ \\
\hline TWA & $\overline{0}$ & $\begin{array}{l}\text { Theorim Acion wil na be } \\
\text { performed. }\end{array}$ \\
\hline NA & 0 & $\begin{array}{l}\text { Theorim Action will nol bo } \\
\text { portormod. }\end{array}$ \\
\hline TNA & $\overline{0}$ & $\begin{array}{l}\text { Interim Action wil nol bo } \\
\text { portormod. }\end{array}$ \\
\hline TWA & 0 & $\begin{array}{l}\text { Intorim Action wil nol be } \\
\text { portormed }\end{array}$ \\
\hline
\end{tabular}

Cost Account Number $3 \times A 06 D 000$

\begin{tabular}{|c|c|c|c|c|c|c|c|c|c|c|c|c|}
\hline \multirow{2}{*}{\multicolumn{2}{|c|}{ Cost Account Tille }} & \multicolumn{2}{|c|}{ OU 2-13 Assessment } & \multicolumn{9}{|c|}{ Cost Account Number $3 \times A 06 D 000$} \\
\hline & & \multirow[b]{2}{*}{ 2. WAGOU } & \multirow[b]{2}{*}{ 4. Mibstione Tito } & \multirow{2}{*}{$\begin{array}{l}\text { 5. Hammock } \\
\text { Duration (Woeks) }\end{array}$} & \multirow{2}{*}{$\begin{array}{l}\text { B. Basotine } \\
\text { Finish Dase }\end{array}$} & \multicolumn{2}{|c|}{ Prerequisite } & \multicolumn{2}{|c|}{ Succossor } & \multirow{2}{*}{$\begin{array}{l}\text { 11. Complation } \\
\text { Classitication }\end{array}$} & \multirow{2}{*}{$\begin{array}{l}\text { 12. Schodulo } \\
\text { Albk } \\
\text { (Weeks) }\end{array}$} & \multirow{2}{*}{$\begin{array}{l}\text { 13. Basellhe Deviation } \\
\text { Basia }\end{array}$} \\
\hline 1. Wos Numbor & 2.10No. & & & & & 7. WBS Number & $8.10 \mathrm{No}$ & 9. WaS Nurrber & 10. $10 \mathrm{No}$ & & & \\
\hline 1.1.2.1.1.D.1 & 37 & $2 \cdot 13$ & $\begin{array}{l}\text { Diall SOW Subrixed lo } \\
\text { EPMDHWW }\end{array}$ & 0.4 & $018 / 80$ & 1.1.2.1.1.4. & $5 A$ & 1.1.2.1.1.0.1 & 30 & 0 & 0 & $\overline{\mathbf{N A}}$ \\
\hline 1.1.2.1.1.D.1 & 30 & $\overline{2-13}$ & $\begin{array}{l}\text { Subrrid Drah Work Plan to } \\
\text { EPMDHWW }\end{array}$ & 21.1 & $11 / 18 / 96$ & 1.1.2.1.1.0.1 & 37 & 1.1.2.1.1.6.2 & 24 & 0 & 0 & $\overline{N A}$ \\
\hline 1.1.2.1.1.0.2 & 24 & $2-13$ & 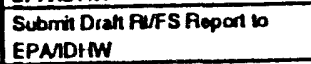 & 70.0 & 6/25/98 & 1.1.2.1.1. .1 & 30 & $\begin{array}{l}1.1 .2 .1 .1 .0 .3 \\
1.1 .10 .1 .1 .1 .2\end{array}$ & $\begin{array}{l}10 \\
30\end{array}$ & 0 & 0 & $\overline{\text { NA }}$ \\
\hline 1.1 .1 .1 .0 .3 & 10 & $2 \cdot 13$ & Submi Drah ROD 10 EPAMDHNY & 67.4 & 014109 & 1.1.2.1.1.0.2 & 24 & 1.1 .2 .1 .1 .0 .3 & 23 & $\overline{0}$ & o & $\mathrm{NA}$ \\
\hline & & 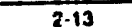 & Final ROO Approved & 22.2 & $11 / 22 / 80$ & 1.1 .2 .1 .1 .0 .3 & 10 & & & 0 & 0 & NA \\
\hline
\end{tabular}

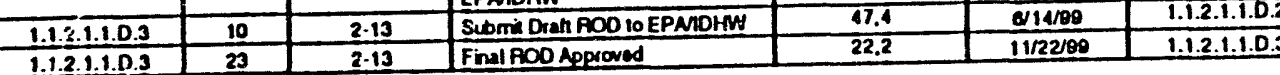

\begin{tabular}{|c|c|c|c|}
\hline Cos: Acce & 116 & \multicolumn{2}{|c|}{ OU 2-04 Assessmenl } \\
\hline \multicolumn{2}{|c|}{ Milessone Relereno } & & \\
\hline 1. Was Number & $2.10 \mathrm{No}$ & 3. WAGOU & 4. Mhertone Tille \\
\hline 1.1.2.1.1.4.6 & 5i & $2-06$ & $\begin{array}{l}\text { Submi Oron Surmmery Report to } \\
\text { EPMDHW }\end{array}$ \\
\hline
\end{tabular}

Cost Account TIIle

\begin{tabular}{|c|c|c|}
\hline & \multirow[b]{3}{*}{ 3. WAGYOU } \\
\hline Mikestone Re & onoe & \\
\hline 1. WES Number & $2.10 \mathrm{No}$ & \\
\hline 1.1 .2 .1 .1 .0 .6 & $2 C$ & 200 \\
\hline 1.1 .2 .1 .1 .0 .6 & 188 & $2-\infty$ \\
\hline 1.1 .2 .1 .1 .8 .0 & 188 & $2-\infty$ \\
\hline
\end{tabular}

Cost Account Number $3 \times A 014000$

\begin{tabular}{|c|c|c|c|c|c|c|c|c|}
\hline \multirow{3}{*}{$\begin{array}{l}\text { 5. Hammock } \\
\text { Duration (Woekg) }\end{array}$} & & \multicolumn{4}{|c|}{ Cost Accoun } & \multirow{3}{*}{$\begin{array}{l}\text { 11. Complotion } \\
\text { Classification }\end{array}$} & \multirow{3}{*}{$\begin{array}{l}\text { 12. Schodule } \\
\text { Pisk } \\
\text { (Weeks) }\end{array}$} & \multirow{3}{*}{$\begin{array}{l}\text { 13. Basolino Doviation } \\
\text { Basis }\end{array}$} \\
\hline & \multirow{2}{*}{$\begin{array}{l}\text { C. Baselino } \\
\text { Finish Dal0 }\end{array}$} & Prorequis & & Succes: & & & & \\
\hline & & 7. WaS Nuriber & $0.10 \mathrm{No}$ & O. WaS Numbor & 10. $10 \mathrm{No}$. & & & \\
\hline 01.4 & $1 / 31 / 05$ & & & 1.1.2.1.1.0.1 & 37 & 0 & 0 & NA \\
\hline
\end{tabular}

Cost Account Number $3 \times A 014000$

\begin{tabular}{|c|c|}
\hline 4. Milostona Tillo & $\begin{array}{l}\text { 5. Hammock } \\
\text { Dusation (Wooks) }\end{array}$ \\
\hline $\begin{array}{l}\text { Oiah SOW Subritod to } \\
\text { EPAMDHW }\end{array}$ & 4.2 \\
\hline Submi Dran ROD Io EPMIDHW & 39.2 \\
\hline Find AOD Approved & 17.2 \\
\hline
\end{tabular}

\begin{tabular}{|c|c|c|c|c|c|c|}
\hline \multicolumn{2}{|c|}{ Prorequisite } & \multicolumn{2}{|c|}{ Successor } & \multirow{2}{*}{$\begin{array}{l}\text { 11. Complation } \\
\text { Classilication: }\end{array}$} & \multirow{2}{*}{$\begin{array}{l}\text { 12. Schodulo } \\
\text { Risk } \\
\text { (Wooks) }\end{array}$} & \multirow{2}{*}{$\begin{array}{l}\text { 13. Basoline Deviation } \\
\text { Bassis }\end{array}$} \\
\hline 7. WaS Muntoor & $0.10 \mathrm{No}$. & O. WaS Number & 10. $10 \mathrm{No}$ & & & \\
\hline 极 & 1 & 1.1.2.1.1.8.6 & 168 & N/A & 0 & $\begin{array}{l}\text { Interim AC-at will not bo } \\
\text { portormed. }\end{array}$ \\
\hline 1.1 .2 .1 .1 .0 .8 & $\mathbf{2 C}$ & 1.1 .2 .1 .1 .8 .8 & 188 & $\mathrm{NA}$ & 0 & Interim Action wil nol be \\
\hline 1.1 .2 .1 .1 .0 .6 & 168 & 1.1 .2 .2 .000 & 450 & NA & 0 & $\begin{array}{l}\text { Thlortm Adion will not bo } \\
\text { pertormed. }\end{array}$ \\
\hline
\end{tabular}




\section{Schedule Contingency Worksheet Waste Area Group 2}

Page 2 of 3

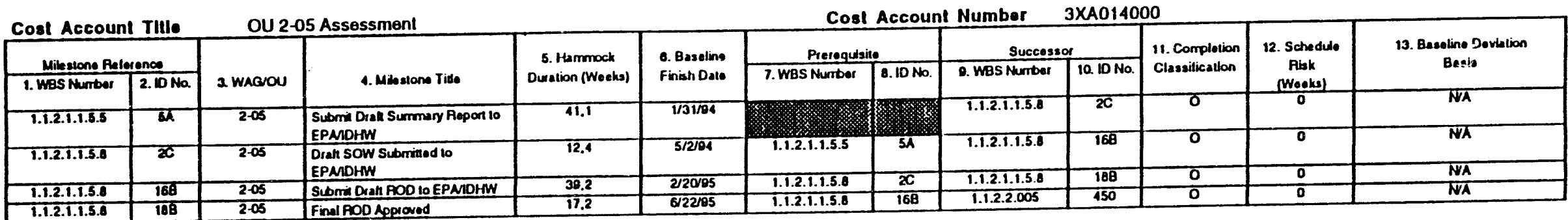

\begin{tabular}{llll}
\hline & OU 2-07 Assessment & Cost Account Number $3 \times A 014000$
\end{tabular}

\begin{tabular}{|c|c|c|c|c|c|c|c|c|c|c|c|c|}
\hline \multirow{2}{*}{\multicolumn{2}{|c|}{ Cost Account Tille }} & \multicolumn{2}{|c|}{ OU 2-07 Assessment } & \multicolumn{9}{|c|}{ Cost Account Number $3 \times A 014000$} \\
\hline & & \multirow[b]{2}{*}{ 3. WAGIOU } & \multirow[b]{2}{*}{ 4. Milostono Titus } & \multirow{2}{*}{$\begin{array}{l}\text { 5. Hanmock } \\
\text { Duration (Woots) }\end{array}$} & \multirow{2}{*}{$\begin{array}{l}\text { 6. Basoline } \\
\text { Finish Dalo }\end{array}$} & \multicolumn{2}{|c|}{ Pierequisilo } & \multicolumn{2}{|c|}{ Successor } & \multirow{2}{*}{$\begin{array}{l}\text { 11. Complotion } \\
\text { Classitication }\end{array}$} & \multirow{2}{*}{$\begin{array}{l}\text { 12. Schodulo } \\
\text { Pisk } \\
\text { (Woeks) }\end{array}$} & \multirow{2}{*}{$\begin{array}{l}\text { 13. Basoline Doviation } \\
\text { Basio }\end{array}$} \\
\hline 1. Was Number & $2.10 \mathrm{Na}$ & & & & & 7. WOS Nurrtber & 6. ID No. & D. WOS Nurtber & 10. $10 \mathrm{No}$. & & & \\
\hline 1.1.2.1.1.7.5 & BA & $2-07$ & Submi Diah Surmary Repon to & $\overline{41.1}$ & 1/3i/94 & & & 1.1.2.1.1.7.7 & $2 \mathrm{C}$ & 0 & 0 & NA \\
\hline 1.1 .2 .1 .1 .7 .7 & $\overline{\mathbf{x C}}$ & 2.07 & \begin{tabular}{|l} 
EPADPHW \\
Diah SOW Submined to
\end{tabular} & 12.4 & 5/2/04 & 1.1 .2 .1 .1 .7 .5 & 5 & 1.1.2.1.1.7.7 & 168 & 0 & 0 & Wh \\
\hline & & & EPMDHW & 39.2 & $2 / 2005$ & T.1.2.1.1.7.7 & $x$ & 1.1 .2 .1 .1 .7 .7 & 188 & o & 0 & WA \\
\hline 1.1.2.1.1.7.7 & 168 & $\frac{2-07}{2-07}$ & SLbmi Dian FOO lo EPMMDHW & $\frac{0.6}{17.2}$ & $6 / 22 / 05$ & 1.1.2.1.1.7.7 & 168 & 1.1.2.2.007 & 750 & 0 & $\overline{0}$ & NA \\
\hline
\end{tabular}

\begin{tabular}{lll}
\hline & Cost Account Mumbor 1.1 .2 .2 .05
\end{tabular}

\begin{tabular}{|c|c|c|c|c|c|c|c|c|c|c|c|c|}
\hline \multirow{2}{*}{\multicolumn{2}{|c|}{ Coul Account Tillo }} & \multicolumn{2}{|c|}{ OU2-05 Hol Wasle Tanks } & \multicolumn{9}{|c|}{ Cost Account Numbor $\quad 1.12 .2 .05$} \\
\hline & & \multirow{2}{*}{ 3. WaGou } & \multirow{2}{*}{ 4. Mibstore Tiste } & \multirow{2}{*}{$\begin{array}{l}\text { 5. Hammock } \\
\text { Duration (Woeks) }\end{array}$} & \multirow{2}{*}{$\begin{array}{l}\text { 6. Basoline } \\
\text { Finish Dale }\end{array}$} & \multicolumn{2}{|c|}{ Prarequisine } & \multicolumn{2}{|c|}{ Successor } & \multirow{2}{*}{$\begin{array}{l}\text { 11. Complotion } \\
\text { Classatication }\end{array}$} & \multirow{2}{*}{$\begin{array}{l}\text { 12. Schodulo } \\
\text { Pisk } \\
\text { (Weeks) }\end{array}$} & \multirow{2}{*}{$\begin{array}{l}\text { 13. Basoline Doviation } \\
\text { Basis }\end{array}$} \\
\hline$\frac{\text { Muks sone He }}{\text { 1. Wos Number }}$ & 2. DONa. & & & & & 7. WES Numbor & 6. $10 \mathrm{No}$ & 9. WOS Nurrber & 10. $10 \mathrm{Na}$ & & & \\
\hline 1.1 .2 .2 .005 & 450 & 2.05 & Submi Drah SOW/AD Wodk Plan & 4.2 & $7 / 25 / 05$ & 1.1 .2 .1 .1 .5 .5 & 5A & $\begin{array}{l}\text { T.1.2.2.005 } \\
\text { 1.1.2.2.005 }\end{array}$ & $\begin{array}{l}108 \\
213\end{array}$ & 0 & 0 & TVA \\
\hline 1.1 .2 .2 .006 & 100 & 2.05 & $\begin{array}{l}10 \text { EPMDHWW } \\
\text { Suburrit Drath Design } 10 \text { EPMDOHW }\end{array}$ & 10.0 & $12 / 1 / 195$ & 1.1 .2 .2 .005 & $\begin{array}{l}450 \\
213\end{array}$ & $\begin{array}{l}1.1 .2 .2 .005 \\
1.1 .2 .2 .005\end{array}$ & $\begin{array}{l}617 \\
017\end{array}$ & 0 & 0 & NA \\
\hline 1.1 .2 .2 .005 & 213 & $2-05$ & $\begin{array}{l}\text { Submin Dran fu Work Plan to } \\
\text { EPAMDHW }\end{array}$ & 10.0 & $12 / 11 / 105$ & $\frac{1.1 .2 .2000}{1.1 .2 .2 .005}$ & $\frac{210}{450}$ & $\begin{array}{l}1.1 .2 .2 .005 \\
1.1 .2 .2 .005 \\
1.1 .2 .2 .005\end{array}$ & $\begin{array}{l}017 \\
617 \\
017 \\
108\end{array}$ & 0 & 0 & NA \\
\hline 1.1 .2 .2 .005 & 617 & 2.05 & Subrin Drafl Pu Repont to & 78.1 & 711007 & $\begin{array}{l}1.1 .2 .2 .005 \\
1.1 .2 .2 .005\end{array}$ & $\begin{array}{l}108 \\
213\end{array}$ & & , & 0 & 0 & Wh \\
\hline $\mathbf{1 . 1 . 2 . 2 . 0 0 6}$ & 017 & $2-05$ & $\begin{array}{l}\text { EPMDOHW } \\
\text { Submix Drath OsM Foport to }\end{array}$ & 82.2 & 8/8197 & $\begin{array}{l}1.1 .2 .2 .005 \\
1.1 .2 .2 .005\end{array}$ & $\begin{array}{l}108 \\
213\end{array}$ & & & $\overline{0}$ & 0 & Nh \\
\hline
\end{tabular}

Cost Account TIIl. OU2-07Cooling Tower/Basins

\begin{tabular}{|c|c|c|c|c|c|c|c|c|c|c|c|c|}
\hline \multirow{2}{*}{\multicolumn{2}{|c|}{ Cost Account Tille }} & \multicolumn{4}{|c|}{ OU2-07Cooling Tower/Basins } & \multicolumn{4}{|c|}{ Cost Account Number } & \multirow{3}{*}{$\begin{array}{l}11 . \text { Completion } \\
\text { Clossilication }\end{array}$} & \multirow{3}{*}{$\begin{array}{l}\text { 12. Schedule } \\
\text { Fisk } \\
\text { Woeks) }\end{array}$} & \multirow{3}{*}{$\begin{array}{l}\text { 13. Basoline Deviation } \\
\text { Bessie }\end{array}$} \\
\hline & & \multirow[b]{2}{*}{3 WAGNOU } & \multirow[b]{2}{*}{ 4. Mistone These } & \multirow{2}{*}{$\begin{array}{l}\text { 5. Hammock } \\
\text { Dusation (Weaks) }\end{array}$} & \multirow{2}{*}{$\begin{array}{l}\text { 6. Basolino } \\
\text { Finbh Dase }\end{array}$} & \multicolumn{2}{|c|}{ Preroquisite } & \multicolumn{2}{|c|}{ Succossoo } & & & \\
\hline 1. Wes Nerriber & $2.10 \mathrm{Na}$ & & & & & 7. WBS Numitor & 8.10 No. & D. WaS Nurrtiol & 10. $10 \mathrm{Na}$ & & & \\
\hline 1.1.2.2.007 & 450 & $2-07$ & $\begin{array}{l}\text { Sibmi Drak SOWfRO Woak Plan } \\
\text { to EPMDHW }\end{array}$ & 4.2 & $7 / 25 / 05$ & 1.1 .2 .1 .1 .7 .7 & 188 & $\begin{array}{l}1.1 .2 .2 .007 \\
1.1 .2 .2 .007\end{array}$ & $\begin{array}{l}106 \\
213\end{array}$ & 0 & 0 & NA \\
\hline 1.1 .2 .2 .007 & 100 & $2-07$ & Submit Dreh Dosigon lo EPMDHWW & 19.0 & $12 / 11 / 95$ & $\begin{array}{l}1.1 .2 .2 .007 \\
1.1 .2 .2 .007\end{array}$ & $\begin{array}{l}1500 \\
213\end{array}$ & $\begin{array}{l}1.1 .2 .2 .007 \\
1.1 .2 .2007\end{array}$ & $\begin{array}{l}817 \\
017\end{array}$ & 0 & $\overline{0}$ & NA \\
\hline \$.1.2.2.007 & 213 & $2-07$ & $\begin{array}{l}\text { Subrit Drath Fu Work Plan to } \\
\text { EPMDOFW }\end{array}$ & 19.0 & $12 / 11 / 105$ & 1.1.2.2.007 & 150 & $\begin{array}{l}1.1 .2 .2 .007 \\
1.12 .2 .007 \\
1.1 .2 .2 .007\end{array}$ & $\begin{array}{l}617 \\
917 \\
108\end{array}$ & 0 & 0 & $\mathbf{N A}$ \\
\hline 1.12 .2 .007 & 617 & 2.07 & 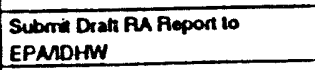 & 73.4 & Q/2017 & $\begin{array}{l}1.1 .2 .2 .007 \\
1.1 .2 .2 .007\end{array}$ & $\begin{array}{l}190 \\
213\end{array}$ & & & 0 & 0 & Nh \\
\hline 1.12 .2 .007 & 817 & $2-07$ & $\begin{array}{l}\text { Submi Dian OSM Repon to } \\
\text { EPMDHWW }\end{array}$ & 78.0 & $7 / 8197$ & $\begin{array}{l}1.1 .2 .2 .007 \\
1.1 .2 .2 .007\end{array}$ & $\begin{array}{l}108 \\
213\end{array}$ & & 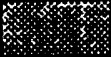 & 0 & 0 & NA \\
\hline
\end{tabular}




\section{Schedule Contingency Worksheet}

Waste Area Group 2

Cost Account TIII0

OU2-10 Warm Wasle Pond Inlerim Aclion

Cost Account Number

Page 3 ol 3

\begin{tabular}{|c|c|c|c|c|c|c|c|c|c|c|c|c|}
\hline 1. Was Nuntbar & $2.10 \mathrm{No}$ & 3. WAGNOU & 4. Minstane Trate & $\begin{array}{l}\text { 5. Mammock } \\
\text { Dustion (Wooks) }\end{array}$ & $\begin{array}{l}\text { 6. Basalino } \\
\text { Finsth Dalo }\end{array}$ & 7. Was Nurrtber & $0.10 \mathrm{No}$ & 0. Was Number & 10. DNo. & $\begin{array}{l}\text { 11. Complation } \\
\text { Classilicalion }\end{array}$ & $\begin{array}{l}\text { 12. Schodulo } \\
\text { Piok } \\
\text { (Weake) }\end{array}$ & $\begin{array}{l}\text { 13. Basoline Devistion } \\
\text { Basto }\end{array}$ \\
\hline 1.12 .2 .010 & 817 & $2 \cdot 10$ & $\begin{array}{l}\text { Submi Diah Pu Flepon to } \\
\text { EPMDHW }\end{array}$ & 40.3 & $3 / 31 / 04$ & & & & & 0 & 0 & NA \\
\hline
\end{tabular}




\section{Schedule Contingency Worksheet Waste Area Group 3}

Page 1 of 1

\begin{tabular}{|c|c|c|c|c|c|c|c|c|c|c|c|c|}
\hline \multicolumn{2}{|c|}{ Cost Account Tille } & \multicolumn{4}{|c|}{ OU-13 WAG 3 Comprehensive RIFS } & \multicolumn{3}{|c|}{ Cost Account Number } & 4113 & \multirow{3}{*}{$\begin{array}{l}\text { 11. Completion } \\
\text { Ctessitication }\end{array}$} & \multirow{3}{*}{$\begin{array}{l}\text { 12. Schodule } \\
\text { Risk } \\
\text { Woeke) }\end{array}$} & \multirow{3}{*}{$\begin{array}{l}\text { 13. Basolino Doviation } \\
\text { Besle }\end{array}$} \\
\hline \multicolumn{2}{|c|}{ Mingrono Polerences } & \multirow[b]{2}{*}{3 WhaOU } & \multirow[b]{2}{*}{ 4. Minstione Tide } & \multirow{2}{*}{$\begin{array}{l}\text { 5. Harmock } \\
\text { Duration (Weoke) }\end{array}$} & \multirow{2}{*}{$\begin{array}{l}\text { 6. Besoline } \\
\text { Finish Dale }\end{array}$} & \multicolumn{2}{|c|}{ Provequisathe } & \multicolumn{2}{|c|}{ Succenssor } & & & \\
\hline 1. Was Nunter & 2.10No. & & & & & 7. WOS Nurtber & C.1DNo. & D. WeS Number & $10.10 \mathrm{No}$. & & & \\
\hline 1.1.3.1.13 & $\infty$ & $2-13$ & $\begin{array}{l}\text { Submid Dren SOW bo } \\
\text { Whap Prgect Managen }\end{array}$ & 15.0 & $2 / 1 / 105$ & 1.1.3.1.7 & $\overline{\omega O H}$ & 1.1.3.1.13 & $\infty$ & 0 & 0 & No \\
\hline 0.1.3.1.13 & $\infty$ & $3-13$ & 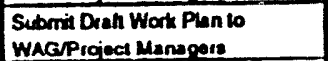 & 20.0 & $1 / 1 / 100$ & 1.1.3.1.13 & $\infty$ & 1.1.3.1.13 & ons & 0 & 0 & Nh \\
\hline 1.1.2.1.13 & aw & $3-13$ & 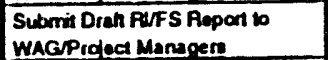 & 83.2 & 9/1907 & 1.1.3.1.13 & $\infty 01$ & $\begin{array}{l}1.1 .3 .1 .13 \\
1.1 .10 .1 .1 .1 .2\end{array}$ & $\begin{array}{c}\text { OSS } \\
30\end{array}$ & 0 & 0 & NA \\
\hline 1.1 .3 .1 .13 & oss & 3.13 & $\begin{array}{l}\text { Subrrid Dian AOO bo WAGPPlojed } \\
\text { Managers }\end{array}$ & 41.1 & 711 red & 1.1.3.1.13 & $\mathbf{W N}$ & I.1.3.1.13 & FF & 0 & 0 & WW \\
\hline 1.1.3.1.13 & FF & $3-13$ & Final FOO Aocepled & 17.0 & 1003000 & 1.1 .3 .1 .13 & oss & $a$ & & 은 & 0 & Nh \\
\hline \multicolumn{2}{|c|}{ Cost Account Tillo } & \multicolumn{2}{|c|}{ WAG 3 Track 2 OUs } & & & \multicolumn{3}{|c|}{ Cost Account Number } & 4109 & & & \\
\hline \multicolumn{2}{|c|}{ Mile stono Relerence } & \multirow[b]{2}{*}{ 2. WAGNOU } & \multirow[b]{2}{*}{ 4. Mindione Ituto } & \multirow{2}{*}{$\begin{array}{l}\text { 5. Hammock } \\
\text { Duration (Wooke) }\end{array}$} & \multirow{2}{*}{$\begin{array}{l}\text { O. Basolino } \\
\text { Fintho Dato }\end{array}$} & \multicolumn{2}{|c|}{ Prosequisite } & \multicolumn{2}{|c|}{ Successor } & \multirow{2}{*}{$\begin{array}{l}\text { 11. Complation } \\
\text { Clasesilicetion }\end{array}$} & \multirow{2}{*}{$\begin{array}{l}\text { 12. Schodulo } \\
\text { Pliok } \\
\text { (Woeke) }\end{array}$} & \multirow{2}{*}{$\begin{array}{l}\text { 13. Bescaline Doviation } \\
\text { Beats }\end{array}$} \\
\hline 1. WaS Merrber & 2.10 Na & & & & & 7. WBS Number & $6.10 \mathrm{Nb}$ & O. WBS Number & 10. DNo. & & & \\
\hline T.1.3.1.7 & क्म & $3-00$ & $\begin{array}{l}\text { Submin Drah Surmmery Report } 10 \\
\text { EPAMOHW } \\
\text { Lest } \alpha \text { Tiack I's and 2's) }\end{array}$ & 82.1 & $4 / 4 / 05$ & & & 1.1.3.1.13 & $\bar{\infty}$ & 0 & 0 & Wh \\
\hline
\end{tabular}

Disconnects:

MK has the following actions in the baseline which are not supported by the WINCO baseline:

OU 3-06 Inlerim Action

OU 3-07 Inlerim Aclion

OU 3-08 Inlerim Aclion

OU 3-13 Final Remediation of WAG 3 
Schedule Contingency Worksheet Waste Area Group 4

Page 1 of 1

\begin{tabular}{|c|c|c|c|c|c|c|c|c|c|c|c|c|}
\hline \multirow{3}{*}{\multicolumn{2}{|c|}{$\frac{\text { Cost Account tillo }}{\text { Mibstono Poloctence }}$}} & \multirow{2}{*}{\multicolumn{2}{|c|}{ WAG 4 Assessment }} & \multicolumn{9}{|c|}{ Cost Account Number $3 \times C A 00000$} \\
\hline & & & & \multirow{3}{*}{$\begin{array}{l}\text { 5. Hammock } \\
\text { Duration (Woeks) }\end{array}$} & \multirow{3}{*}{$\begin{array}{l}\text { 6. Basoline } \\
\text { Finish Date }\end{array}$} & \multirow{2}{*}{\multicolumn{2}{|c|}{ Prorequisine }} & \multirow{2}{*}{\multicolumn{2}{|c|}{ Successor }} & \multirow{3}{*}{$\begin{array}{l}\text { 11. Complotion } \\
\text { Clessalication }\end{array}$} & \multirow{3}{*}{$\begin{array}{l}\text { 12. Schodulb } \\
\text { Piskt } \\
\text { (Weato) }\end{array}$} & \multirow{3}{*}{$\begin{array}{l}\text { 13. Basedine Doviation } \\
\text { Bests }\end{array}$} \\
\hline & & \multirow[b]{2}{*}{ 3. WAGNOU } & \multirow[b]{2}{*}{ 4. Misostone Tivio } & & & & & & & & & \\
\hline 1. WaS Mumber & $2.10 \mathrm{Na}$ & & & & & 7. WES Nurrbor & 6. $10 \mathrm{No}$. & 9. WBS Nurrber & 10. 10 No. & & & \\
\hline 1.1.4.1.1.1.2 & $\overline{a r}$ & 1.05 & $\begin{array}{l}\text { Scbmil Final Summay Report io } \\
\text { EPMDHW }\end{array}$ & 8.1 & $2 / 23 / 00$ & & & 1.1 .4 .1 .1 .1 .8 & 37 & 0 & 0 & NA \\
\hline T.1.1.1.1.1A & 10 & $4 \cdot 12$ & $\begin{array}{l}\text { SLbmil Diald AOO end FS to } \\
\text { EPMDHW }\end{array}$ & 52.3 & $7117 / 85$ & 1.1 .1 .1 .1 .1 .0 & $\infty 0$ & 1.1.4.1.1.1. & 23 & ס & 0 & Wh \\
\hline 1.1.4.1.1.1. & $\infty$ & 4-12 & 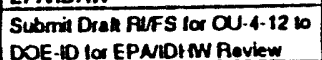 & 01,2 & $6 / 2394$ & & & $\begin{array}{l}1.1 .4 .1 .1 .1 .4 \\
\text { 1.1.4.1.1.1.8 }\end{array}$ & $\begin{array}{l}10 \\
30\end{array}$ & 0 & 0 & NA \\
\hline 1.1.4.1.1.1A & 23 & $9-12$ & Final HOD Aocepled & 20.0 & $12 / 6 / 05$ & 1.1.4.1.1.1. & 10 & & & 0 & 0 & Twa \\
\hline 1.1.6.1.1.1.8 & 37 & $4-13$ & $\begin{array}{l}\text { Drah SOW Submited to } \\
\text { EPMDEHAt }\end{array}$ & 18.1 & 81000 & 1.1.4.1.1.1.2 & ल & 1.1.4.1.1.1.8 & 30 & 0 & 0 & NA \\
\hline 1.1.4.1.1.1.8 & 30 & $4 \cdot 13$ & $\begin{array}{l}\text { Submit Dran Work Plan to } \\
\text { EPMIDHW }\end{array}$ & 21.1 & 11/10/08 & 1.1.4.1.1.1. B & 37 & 1.1.4.1.1.1.C & 24 & 0 & 0 & N/A \\
\hline T.1.4.1.1.1.C & 24 & 4.13 & $\begin{array}{l}\text { Subrrit Dinh Falfs Repon bo } \\
\text { EPMDHW }\end{array}$ & 70.0 & $\$ 25 / 98$ & 1.1.4.1.1.1.8 & 30 & $\begin{array}{l}1.1 .10 .1 .1 .1 .2 \\
1.1 .4 .1 .1 .1 .0\end{array}$ & $\begin{array}{l}30 \\
10\end{array}$ & 0 & $\overline{0}$ & NA \\
\hline 1.1.4.1.1.1. & 10 & $4-13$ & Submi Dran ROO 10 EPAMDHW & 54.4 & $8 / 390$ & 1.1.4.1.1.1.C & 24 & 1.1.4.1.1.1. & 23 & 0 & 0 & Wh \\
\hline 1.1.4.1.1.1. & 23 & $4 \cdot 13$ & Final ROO Appioved & 22.1 & $1 / 18 / 00$ & 1.1 .4 .1 .1 .1 .0 & 10 & 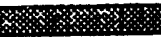 & & 0 & 0 & NR \\
\hline
\end{tabular}

Disconnects:

MK has the lollowing actions in the baseline that are nol supported from the WAG 4 baseline:

OU 4-05A Interim Action

OU 4.05B Interim Action

OU 4-06A Interim Action

OU 4-06B intorim Aclion

OU 4-67A Inlerim Action

OU 4.07B Interim Action

OU 4-08 Interim Aclion

OU 4-09 Interim Action

OU 4-09A Inlerim Action

OU 4-09B Interim Action

OU 4.010 Interim Action

OU 4-12A Interim Action 


\section{Schedule Contingency Worksheet}

Waste Area Group 5

\begin{tabular}{|c|c|c|c|c|c|c|c|c|c|c|c|c|}
\hline \multicolumn{2}{|c|}{ Cost Account Tillo } & \multicolumn{2}{|c|}{ WAG 5 Comprehensive RI/FS } & \multicolumn{9}{|c|}{ Cost Account Number $3 \times B A 30000$} \\
\hline Milestone Pal & rences & \multirow[b]{2}{*}{ 3. WAGNOU } & \multirow[b]{2}{*}{ 4. Mioslono Tivilo } & \multirow{2}{*}{$\begin{array}{l}\text { 5. Hammock } \\
\text { Durntion (Weoks) }\end{array}$} & \multirow{2}{*}{$\begin{array}{l}\text { O. Basoline } \\
\text { Finbeh Dase }\end{array}$} & \multicolumn{2}{|c|}{ Piolequisite } & \multicolumn{2}{|c|}{ Successor } & \multirow{2}{*}{$\begin{array}{l}\text { 11. Complation } \\
\text { Classilication }\end{array}$} & \multirow{2}{*}{$\begin{array}{l}\text { 12. Schodule } \\
\text { Aisk } \\
\text { Wooks) }\end{array}$} & \multirow{2}{*}{$\begin{array}{l}\text { 13. Basolino Dovistion } \\
\text { Basis }\end{array}$} \\
\hline 1. WoS Muntbet & 2.10Na. & & & & & 7. WBS Numbor & B. 10 No. & O. WBS Nurrber & 10. 10 No. & & & \\
\hline 1.1.5.1.1.3.1 & 37 & $5-12$ & $\begin{array}{l}\text { Drent SOW Subminod to } \\
\text { EPMDOHW }\end{array}$ & 20.1 & $8 / 2 / 90$ & 1.1.5.1.1.2.4 & 103 & 1.1.5.1.1.3.1 & 30 & 0 & 0 & NA \\
\hline 1.1.5.1.1.3.1 & 30 & $5-12$ & $\begin{array}{l}\text { Sutorit Dinh Work Plan to } \\
\text { EPMDHWW }\end{array}$ & 17.2 & $12 / 8 / 08$ & 1.1.5.1.1.3.1 & $\overline{37}$ & 1.1 .5 .1 .1 .3 .2 & 24 & $\overline{0}$ & 0 & WA \\
\hline 1.1 .5 .1 .1 .3 .2 & 24 & 5-12 & $\begin{array}{l}\text { Submil Dian FaAfS Report b } \\
\text { EPMOHW }\end{array}$ & 81.1 & $7 / 30 / 98$ & T.1.5.1.1.3.1 & 30 & $\begin{array}{l}1.1 .10 .1 .1 .1 .2 \\
1.1 .5 .1 .1 .3 .3 \\
\end{array}$ & $\begin{array}{l}30 \\
10\end{array}$ & O & $\overline{0}$ & NA \\
\hline $1.1 .5 \cdot 1.1 .3 .3$ & 10 & 5.12 & SLOmi Diah FOD 10 EPAIDHWW & 42.4 & 011199 & 1.1 .5 .1 .1 .3 .2 & 24 & 1.1 .5 .1 .1 .3 .3 & 23 & 0 & 0 & NA \\
\hline & & & Final ROO Approved & 17.1 & $10 / 13 / 80$ & 1.1 .5 .1 .1 .3 .3 & 10 & 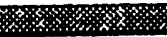 & & 0 & 0 & $\mathrm{NA}$ \\
\hline
\end{tabular}

\begin{tabular}{|c|c|c|c|c|c|c|c|c|c|c|c|c|}
\hline \multicolumn{2}{|c|}{ Cost Accounl Tille } & \multicolumn{2}{|c|}{ WAG 5 Track 2's } & \multicolumn{9}{|c|}{ Cost Account Number 3XBA20000 } \\
\hline \multicolumn{2}{|c|}{ Milestone Ploterenoe } & \multirow[b]{2}{*}{ 3. WAGOU } & \multirow[b]{2}{*}{ 4. Misostone Tille } & \multirow{2}{*}{$\begin{array}{l}\text { 6. Hammock } \\
\text { Duretion (Weoke) }\end{array}$} & \multirow{2}{*}{$\begin{array}{l}\text { O. Buseline } \\
\text { Finish Dato }\end{array}$} & \multicolumn{2}{|c|}{ Prerequisite } & \multicolumn{2}{|c|}{ Successor } & \multirow{2}{*}{$\begin{array}{l}\text { 11. Complotion } \\
\text { Clessilleation }\end{array}$} & \multirow{2}{*}{$\begin{array}{c}\text { 12. Schodulo } \\
\text { Plbk } \\
\text { (Woeka) }\end{array}$} & \multirow{2}{*}{$\begin{array}{l}\text { 13. Basoline Dovistion } \\
\text { Basls }\end{array}$} \\
\hline 1. Was Nunbet & 2.DNo. & & & & & 7. WBS Number & B. $10 \mathrm{No}$. & D. Was Nuntbor & 10. $10 \mathrm{Na}$ & & & \\
\hline 1.1.5.1.1.2.5 & म & $5-\infty$ & $\begin{array}{l}\text { Submi Final Summary Roport bo } \\
\text { EPMDHFW }\end{array}$ & 62.4 & $3 / 12 / 106$ & & & 1.1.5.1.1.3.1 & 37 & 0 & 0 & NA \\
\hline 1.1.5.1.1.2.3 & 105 & $5-07$ & $\begin{array}{l}\text { Sibmit Final Summary fiopont bo } \\
\text { EPMD+WW }\end{array}$ & 10.4 & $6 / 1603$ & & & $\begin{array}{l}1.1 .5 .1 .1 .3 .1 \\
1.1 .5 .2 .007\end{array}$ & $\begin{array}{l}37 \\
450\end{array}$ & $\pi$ & 4 & $\begin{array}{l}\text { Comploted } 5 / 18 / 93 \\
\text { Ahead a Schodule }\end{array}$ \\
\hline 1.1.5.1.1.2.1 & 100 & $5-05$ & $\begin{array}{l}\text { Submit Fhal Surmman Repont to } \\
\text { EPMDH mW }\end{array}$ & 71.1 & $3 / 15 / 05$ & & & $\begin{array}{l}1.1 .5 .1 .1 .3 .1 \\
1.1 .5 .2 .005\end{array}$ & $\begin{array}{l}37 \\
450\end{array}$ & 0 & 0 & NA \\
\hline 1.1.5.1.1.2.4 & 100 & $5-\infty$ & $\begin{array}{l}\text { Submil Final Surmmary Roport to } \\
\text { EPMDtiw }\end{array}$ & 71.1 & 5/2/08 & & & $\begin{array}{l}1.1 .5 \cdot 1.1 .3 .1 \\
1.1 .5 .2 .008\end{array}$ & $\begin{array}{l}37 \\
450\end{array}$ & 0 & 0 & NA \\
\hline
\end{tabular}

\begin{tabular}{|c|c|c|}
\hline & Mibatone Rolerenos & 3. WAGOU \\
\hline 1.1.5.2.007 & 450 & 5.07 \\
\hline 1.1 .5 .2 .007 & 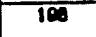 & 5.07 \\
\hline 1.1.5.2.007 & $\overline{213}$ & 5-07 \\
\hline 1.1 .5 .2 .007 & 017 & $5-07$ \\
\hline 1.1.5.2.007 & 017 & 5-07 \\
\hline
\end{tabular}

ARA-IS anilay Sower System Pad mear ARA G27

Cost Account Number 1.1.5.2.07

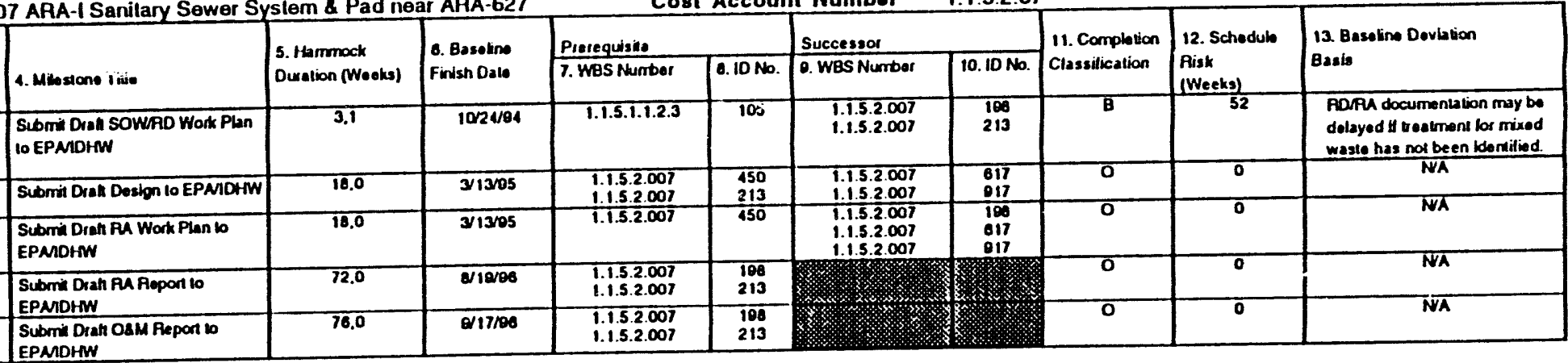




\section{Schedule Contingency Worksheet \\ Waste Area Group 5}

Page 2 of 2

Cost Accouni TIIle OU 5-05 ARA-II SL-1 Burial Ground Cleanup

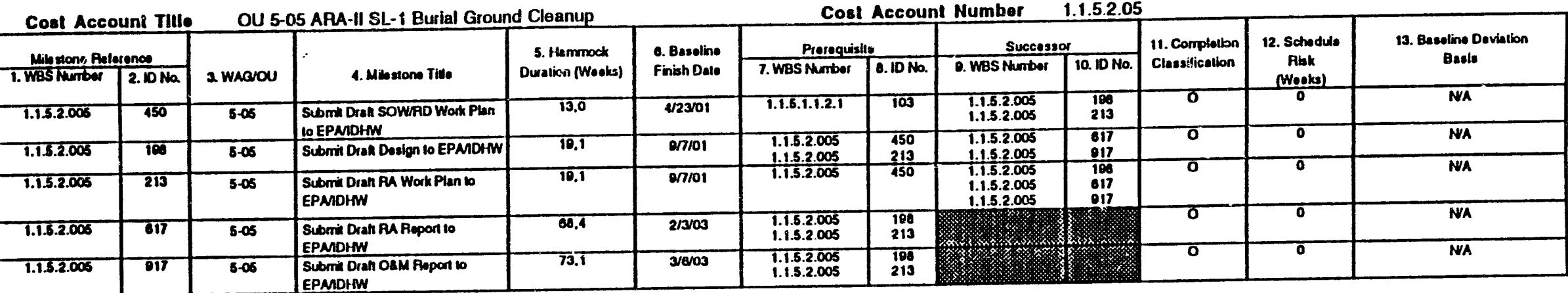

Cosl Accoun! TIIl OU 5-08 PBF Injection Wells/SPEAT I Soepage Pil

Cost Account Number 1.1.5.2.08

\begin{tabular}{|c|c|c|c|c|c|c|c|c|c|c|c|c|}
\hline \multicolumn{2}{|c|}{ Cosl Account Tllle } & \multicolumn{4}{|c|}{ OU 5-08 PBF Injection Wells/SPERT I Soepage Pil } & \multicolumn{4}{|c|}{ Cost Account Number $\quad 1.1 .5 .2 .08$} & \multirow{3}{*}{$\begin{array}{l}\text { 11. Completion } \\
\text { Clesellleallon }\end{array}$} & \multirow{3}{*}{$\begin{array}{l}\text { 12. Schedulo } \\
\text { Flakk } \\
\text { (Woeks) }\end{array}$} & \multirow{3}{*}{$\begin{array}{l}\text { 13. Basolino Doviastion } \\
\text { Basle }\end{array}$} \\
\hline & non & \multirow[b]{2}{*}{ 2. WAGNOU } & \multirow[b]{2}{*}{ 4. Mlosiono Titso } & \multirow{2}{*}{$\begin{array}{c}\text { 5. Hammock } \\
\text { Durstion (Wooks) }\end{array}$} & \multirow{2}{*}{$\begin{array}{l}\text { 6. Baseline } \\
\text { Finteh Date }\end{array}$} & \multicolumn{2}{|c|}{ Prerequisile } & \multicolumn{2}{|c|}{ Successor } & & & \\
\hline 1. Wos Number & $2.10 \mathrm{Na}$ & & & & & 7. WBS Numbor & $0.10 \mathrm{No}$ & O. WOS Nuntor & 10. $1 \mathrm{DNO}$. & & & \\
\hline 1.1.5.2.006 & 450 & 508 & $\begin{array}{l}\text { SLbmin Droh SOW/AD Work Plan } \\
\text { Io EPNIDHW }\end{array}$ & 13.0 & $4 / 23 / 01$ & 1.1.5.1.1.2. & 103 & $\begin{array}{l}\text { T.1.5.2.006 } \\
1.1 .5 .2 .006\end{array}$ & $\begin{array}{l}198 \\
213\end{array}$ & o & $\overline{0}$ & NA \\
\hline 1.1.6.2.000 & 100 & $5-00$ & Submit Drat Dosign to EPMDOHW & 18.1 & 9/101 & $\begin{array}{l}1.1 .5 .2 .000 \\
1.1 .5 .2 .006\end{array}$ & $\begin{array}{l}455 \\
213\end{array}$ & $\begin{array}{l}1.1 .5 .2 .006 \\
1.1 .5 .2 .000\end{array}$ & $\begin{array}{l}817 \\
017\end{array}$ & 0 & $\overline{0}$ & NA \\
\hline 1.15 .2 .000 & 213 & $3-08$ & $\begin{array}{l}\text { Subrim Draht Fu Work Plan lo } \\
\text { EPMDHWW }\end{array}$ & 10.1 & का/101 & 1.1 .5 .2 .000 & $\frac{10}{450}$ & $\begin{array}{l}1.1 .2 .2 .000 \\
\text { 1.1.5.2.006 } \\
1.1 .5 .2 .000\end{array}$ & $\begin{array}{l}180 \\
617 \\
017\end{array}$ & 0 & - & $\overline{N A}$ \\
\hline 1.1 .5 .2 .008 & 817 & $5-00$ & $\begin{array}{l}\text { Submi Dinh Fu Report } 10 \\
\text { EPMDOHW }\end{array}$ & 60.4 & $2 / 3 / 203$ & $\begin{array}{l}\text { 1.1.5.2.008 } \\
\text { 1.1.5.2.000 }\end{array}$ & $\begin{array}{l}100 \\
213\end{array}$ & & & 0 & 0 & NA \\
\hline $\mathbf{1 . 1 . 5 . 2 . 0 0 0}$ & 917 & $5-\infty$ & $\begin{array}{l}\text { Submik Drath OsM Feport bo } \\
\text { EPMDDHW }\end{array}$ & 73.1 & $3 / 6 / 03$ & $\begin{array}{l}1.1 .5 .2 .009 \\
\text { 1.1.5.2.008 } \\
\end{array}$ & $\begin{array}{l}100 \\
213 \\
\end{array}$ & & & $\sigma$ & 0 & N/A \\
\hline
\end{tabular}

Cost Account TIIlo OU 5-13 PBF-8 Corrosive Waste Sump \& PBF-10 Pond

\begin{tabular}{|c|c|c|c|c|c|c|c|c|c|c|c|c|}
\hline \multirow{2}{*}{\multicolumn{2}{|c|}{ Cost Account TIIlo }} & \multicolumn{4}{|c|}{ OU 5-13 PBF-8 Corrosive Wasle Sump \& PBF-10 Pond } & \multicolumn{4}{|c|}{ Cost Account Number $\quad 1.1 .5 .2 .13$} & \multirow{3}{*}{$\begin{array}{l}\text { 11. Completion } \\
\text { Classilication }\end{array}$} & \multirow{3}{*}{$\begin{array}{l}\text { 12. Schedulo } \\
\text { Risk } \\
\text { (Woeks) }\end{array}$} & \multirow{3}{*}{$\begin{array}{l}\text { 13. Basoline Dovistion } \\
\text { Basiz }\end{array}$} \\
\hline & & \multirow[b]{2}{*}{ 3. WAGNOU } & \multirow[b]{2}{*}{ 4. Mibstome Titho } & \multirow{2}{*}{$\begin{array}{l}\text { 5. Hammock } \\
\text { Ouration (Weeks) }\end{array}$} & \multirow{2}{*}{$\begin{array}{l}\text { C. Basoline } \\
\text { Finish Dave }\end{array}$} & \multicolumn{2}{|c|}{ Prenequisite } & \multicolumn{2}{|c|}{ Successor } & & & \\
\hline 1. Wensumber & 2. 10 No. & & & & & 7. WaS Nuriber & 6. ID No. & O. WeS Number & 10.10No. & & & \\
\hline 1.1.3.2.012 & $10 x$ & 5-13 & $\begin{array}{l}\text { Submil ProllPio Final Design lo } \\
\text { EPMDHW }\end{array}$ & 16.0 & 7/23/0s & T.1.5.2.013 & 2iY & $\begin{array}{l}7.1 .5 .2 .013 \\
1.1 .5 .2 .013 \\
1.1 .5 .2 .013 \\
\end{array}$ & $\begin{array}{l}218 \\
017 \\
017\end{array}$ & 8 & 17.0 & $\begin{array}{l}\text { Delay due do sompling and } \\
\text { enalysib elfort }\end{array}$ \\
\hline 1.1.5.2.013 & $21 Y$ & 5.13 & $\begin{array}{l}\text { Submin Deah Fu Woak Plan bo } \\
\text { EPMDDHW }\end{array}$ & 18.1 & 8/\%03 & & & $\begin{array}{l}\text { 1.1.5.2.013 } \\
\text { 1.1.5.2.013 }\end{array}$ & $\begin{array}{l}817 \\
017\end{array}$ & 0 & 0 & NA \\
\hline T.1.5.2.013 & 017 & 5-13 & SLOmi O\&M Fopor EPMDHW & $\overline{51,2}$ & $1 / 22 / 24$ & $\begin{array}{l}1.1 .5 .2 .013 \\
1.1 .5 .2 .013\end{array}$ & $\begin{array}{ll}211 \\
19 x\end{array}$ & & & o & 0 & N/A \\
\hline T.1.6.2.013 & 617 & 5-13 & SGbmi RA Repon lo EPMDHFY & 40.4 & $7 / 20 / 94$ & $\begin{array}{l}1.1 .5 .2 .013 \\
1.1 .5 .2 .013\end{array}$ & $\begin{array}{l}21 Y \\
10 x\end{array}$ & & & o & 0 & NA \\
\hline
\end{tabular}




\section{Schedule Contingency Worksheet \\ Waste Area Group 5}

Page 2 of 2

\begin{tabular}{|c|c|c|c|c|c|c|c|c|c|c|c|c|}
\hline \multicolumn{2}{|c|}{ Cost Account Tille } & \multicolumn{3}{|c|}{ OU 5-05 ARA-11 SL-1 Burial Ground Cleanup } & \multicolumn{5}{|c|}{ Cost Account Number } & \multirow{3}{*}{$\begin{array}{l}\text { 11. Complotion } \\
\text { Classitication }\end{array}$} & \multirow{3}{*}{$\begin{array}{l}\text { 12. Scthedule } \\
\text { Pisk } \\
\text { (Weeks) }\end{array}$} & \multirow{3}{*}{$\begin{array}{l}\text { 13. Baseline Doviation } \\
\text { Basise }\end{array}$} \\
\hline Mikstone Pe & & \multirow[b]{2}{*}{ 3. WhaOU } & \multirow[b]{2}{*}{ 4. Mibstane Tille } & \multirow{2}{*}{$\begin{array}{l}\text { 5. Hanmock } \\
\text { Duration (Wooks) }\end{array}$} & \multirow{2}{*}{$\begin{array}{l}\text { O. Besolino } \\
\text { Finish Dato }\end{array}$} & \multicolumn{2}{|c|}{ Pioregulaine } & \multicolumn{2}{|c|}{ Successor } & & & \\
\hline 1. Was Neriber & $2.10 \mathrm{No}$ & & & & & 7. WaS Number & $0.10 \mathrm{No}$ & O. WaS Nuntior & 10. ID No. & & & \\
\hline 1.1.5.2.005 & 450 & $5-05$ & $\begin{array}{l}\text { Sibmi Drah SoWfro Work Plan } \\
\text { lo EPMDrNy }\end{array}$ & 13.0 & 1/23/01 & 1.1.5.1.1.2.1 & 103 & $\begin{array}{l}\text { T.1.5.2.005 } \\
\text { 1.1.5.2.005 }\end{array}$ & $\begin{array}{l}100 \\
213\end{array}$ & O & 0 & NA \\
\hline 1.1 .5 .2 .005 & 10 & 6.05 & Submit Drat Dosion 10 EPMDHW & 10.1 & 9/7/01 & $\begin{array}{l}1.1 .5 .2 .005 \\
1.1 .5 .2 .005\end{array}$ & $\begin{array}{l}150 \\
213\end{array}$ & $\begin{array}{l}\text { T.1.5.2.005 } \\
\text { 1.1.5.2.005 }\end{array}$ & $\begin{array}{l}017 \\
017\end{array}$ & 万人 & $\overline{0}$ & NA \\
\hline 1.1.5.2.005 & 213 & $5-05$ & $\begin{array}{l}\text { Submi Dren Fu Work Ptan b } \\
\text { EPADOHW }\end{array}$ & 10.1 & on/01 & 1.1.5.2.005 & 450 & $\begin{array}{l}1.1 .5 .2 .005 \\
1.1 .5 .2 .005 \\
1.1 .5 .2 .005\end{array}$ & $\begin{array}{l}100 \\
617 \\
017\end{array}$ & 0 & 0 & NA \\
\hline $\mathbf{1 . 1 5 . 2 . 0 0 5}$ & 617 & 6.05 & $\begin{array}{l}\text { Subrit Drath PU Flepon lo } \\
\text { EPMDHW }\end{array}$ & 66.4 & 2/3/03 & $\begin{array}{l}1.1 .5 .2 .005 \\
\text { 1.1.5.2.005 }\end{array}$ & $\begin{array}{l}198 \\
213 \\
\end{array}$ & & & o & 0 & $\mathbf{N / A}$ \\
\hline 1.1.5.2.005 & 017 & 5.05 & $\begin{array}{l}\text { Submi Diall O\&M Peport to } \\
\text { EPMDHWW }\end{array}$ & 73.1 & $3 / \sqrt{6 / 03}$ & $\begin{array}{l}\text { 1.1.5.2.005 } \\
\text { i.1.5.2.005 }\end{array}$ & $\begin{array}{l}709 \\
213\end{array}$ & & & $\overline{0}$ & 0 & NA \\
\hline
\end{tabular}

Cost Account Tille OU 5-08 PBF Injection Wells/SPERT I Seepage Pit

\begin{tabular}{|c|c|c|c|c|c|}
\hline \multicolumn{2}{|c|}{ Milestone Puforences } & \multirow[b]{2}{*}{ 3. WAONOU } & \multirow[b]{2}{*}{ 4. Minstone Tillo } & \multirow{2}{*}{$\begin{array}{l}\text { 5. Hammock } \\
\text { Duratlon (Wooks) }\end{array}$} & \multirow{2}{*}{$\begin{array}{l}\text { C. Basolino } \\
\text { Finthe Dato }\end{array}$} \\
\hline 1. Wes Nomber & $2.10 \mathrm{Na}$ & & & & \\
\hline 1.1 .5 .2 .000 & 450 & 500 & $\begin{array}{l}\text { Submi Dran SOW/AD Work Plan } \\
\text { loEPMDPFW }\end{array}$ & 13.0 & 1/23/01 \\
\hline 1.1 .6 .2 .000 & 10 & $5-06$ & Submil Dral Design to EPMDHW & 10.1 & $07 / 101$ \\
\hline 1.1.5.2.000 & 213 & $5-\infty$ & $\begin{array}{l}\text { Submik Diah Fu Work Plan to } \\
\text { EPAMDHW }\end{array}$ & 10.1 & Q/7/01 \\
\hline 1.1.5.2.008 & 617 & $5-\infty$ & $\begin{array}{l}\text { Submi Dralt RA Report to } \\
\text { EPMiDHW }\end{array}$ & 60.4 & $2 / 3 / 03$ \\
\hline 1.1.5.2.000 & 017 & $5-06$ & $\begin{array}{l}\text { Submil Dian OSM Rapon io } \\
\text { EPMIOHW }\end{array}$ & 73.1 & $3 / 6 / 03$ \\
\hline
\end{tabular}

Cost Account Number 1.1.5.2.08

\begin{tabular}{|c|c|c|c|c|c|c|c|c|c|c|c|c|}
\hline \multirow{2}{*}{\multicolumn{2}{|c|}{ Cost Acceunt IIIO }} & \multicolumn{4}{|c|}{ OU 5-13 PBF-B Corrosive Wasle Sump \& PBF-10 Pond } & \multicolumn{3}{|c|}{ Cost Account Number } & 1.1.5.2.13 & \multirow{3}{*}{$\begin{array}{l}\text { 11. Complation } \\
\text { Classitication }\end{array}$} & \multirow{3}{*}{$\begin{array}{l}\text { 12. Schodule } \\
\text { Aisk } \\
\text { (Woeks) }\end{array}$} & \multirow{3}{*}{$\begin{array}{l}\text { 13. Baseline Doviation } \\
\text { Basis }\end{array}$} \\
\hline & & & & 5. Hummock & 6. Basoline & Prerequis: & & Success & & & & \\
\hline 1. Was Numbor & $2.10 \mathrm{He}$ & 2. WAGIOU & 4. Milo stome Tiles & Duration (Weekes) & Finish Dolle & 7. WaS Nembor & o. $10 \mathrm{No}$. & 9. WOS Number & 10.10 No. & & & \\
\hline 1.1.5.2.013 & $10 x$ & 6.13 & $\begin{array}{l}\text { Submit ProuPro Final Design to } \\
\text { EPMDHW }\end{array}$ & 16.0 & $7 / 23 / 03$ & 1.1.5.2.013 & $2 \pi$ & $\begin{array}{l}1.1 .5 .2 .013 \\
1.1 .5 .2 .013 \\
1.1 .5 .2 .013 \\
\end{array}$ & $\begin{array}{l}219 \\
017 \\
617\end{array}$ & 7 & 17.0 & $\begin{array}{l}\text { Dolay due lo eampling and } \\
\text { enalyale oflon }\end{array}$ \\
\hline 1.1 .5 .2 .013 & $\overline{21 Y}$ & 5-13 & $\begin{array}{l}\text { Submi Drafh Pu Work Plan lo } \\
\text { EPMDHW }\end{array}$ & 18.1 & 8/0/03 & & & $\begin{array}{l}\text { 1.1.5.2.013 } \\
1.1 .5 .2 .013\end{array}$ & $\begin{array}{l}617 \\
017\end{array}$ & o & $\overline{0}$ & $\overline{\text { NA }}$ \\
\hline 1.1.5.2.013 & 017 & 5-13 & SLbmi OAM Flepen EPMDHW & 61,2 & $8 / 22 / 04$ & $\begin{array}{l}1.1 .5 .2 .013 \\
1.1 .3 .2 .013\end{array}$ & ivx & & 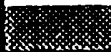 & O & D & N/K \\
\hline 1.1.5.2.013 & 617 & 5.13 & Sibmi PU Repon to EPMIDHWH & 46.4 & $7 / 2004$ & $\begin{array}{l}\text { T.1.5.2.013 } \\
\text { i.1.5.2.013 }\end{array}$ & $\begin{array}{l}21 Y \\
10 x\end{array}$ & 级 & & ర & D & NA \\
\hline
\end{tabular}


Schedule Contingency Worksheet Waste Area Group 6

Page 1 of 1

\begin{tabular}{|c|c|c|c|c|c|c|c|c|c|c|c|c|}
\hline \multirow{2}{*}{\multicolumn{2}{|c|}{$\frac{\text { Cost Account Tille }}{\text { Minstone Peloronos }}$}} & \multicolumn{2}{|c|}{ WAG 6 Assessment } & \multicolumn{5}{|c|}{ Cost Account Numbor } & \multicolumn{3}{|c|}{$3 \times \times A 30000$} & \multirow{3}{*}{$\begin{array}{l}\text { 13. Basoline Dovintion } \\
\text { Besio }\end{array}$} \\
\hline & & \multirow[b]{2}{*}{ 3. WAGNOU } & \multirow[b]{2}{*}{ 4. Mibstone Tite } & \multirow{2}{*}{$\begin{array}{l}\text { 5. Honmock } \\
\text { Duralton (Wooks) }\end{array}$} & \multirow{2}{*}{$\begin{array}{l}\text { O. Basolino } \\
\text { Finish Dale }\end{array}$} & \multicolumn{2}{|c|}{ Prorequisite } & \multicolumn{2}{|c|}{ Successor } & \multirow{2}{*}{$\begin{array}{l}\text { 11. Completion } \\
\text { Classitication }\end{array}$} & \multirow{2}{*}{$\begin{array}{l}\text { 12. Schodulo } \\
\text { Pisk } \\
\text { Weoks) }\end{array}$} & \\
\hline T. WeS Numbor & $2.10 \mathrm{Na}$ & & & & & 7. WBS Number & $0.10 \mathrm{No}$ & O. WaS Nurriber & 10. IO No. & & & \\
\hline 1.1.0.1.1.1.5 & $\bar{M}$ & 8.02 & $\begin{array}{l}\text { Subrix Diath Surmary fieporl 10 } \\
\text { EPMDHW }\end{array}$ & 133,4 & $12 / 7 / 95$ & & & 1.1 .10 .1 .1 .1 .2 & 37 & 0 & 0 & Nh \\
\hline
\end{tabular}


Schedule Contingency Worksheet

Waste Area Group 7

Page 1 of 3

\begin{tabular}{|c|c|c|c|c|c|c|c|c|c|c|c|c|}
\hline \multirow{2}{*}{\multicolumn{2}{|c|}{ Cost Account TIllo }} & \multicolumn{2}{|c|}{ Pad A ROD } & \multicolumn{5}{|c|}{ Cost Account Numbor } & \multicolumn{3}{|c|}{$3 \times w 031000$} & \multirow{3}{*}{$\begin{array}{l}\text { 13. Basoline Deviation } \\
\text { Basls }\end{array}$} \\
\hline & & \multirow[b]{2}{*}{ 3. WAGOU } & \multirow[b]{2}{*}{ 4. Milostone Tallo } & \multirow{2}{*}{$\begin{array}{l}\text { 5. Hammock } \\
\text { Duration (Woeka) }\end{array}$} & \multirow{2}{*}{$\begin{array}{l}\text { C. Beseline } \\
\text { Finish Date }\end{array}$} & \multicolumn{2}{|c|}{ Polegquisile } & \multicolumn{2}{|c|}{ Successor } & \multirow{2}{*}{$\begin{array}{l}\text { 11. Completion } \\
\text { Classitication }\end{array}$} & \multirow{2}{*}{$\begin{array}{l}\text { 12. Schodule } \\
\text { Pisk } \\
\text { (Weaks) }\end{array}$} & \\
\hline 1. WBS Number & 2.10 Na & & & & & 7. WaS Number & $0.10 \mathrm{No}$. & D. WBS Nurribor & 10.10 No. & & & \\
\hline T.1.7.1.1.1.1 & 3 & $7 \cdot 12$ & Transma Drah FOD \& EPMDOHW & Elore TN & 1/7/83 & & & $\begin{array}{l}1.1 .7 .1 .1 .1 .1 \\
1.1 .7 .1 .1 .1 .1\end{array}$ & $\begin{array}{l}31 \\
36\end{array}$ & $\mathbf{T}$ & $\frac{(\log 15}{37}$ & 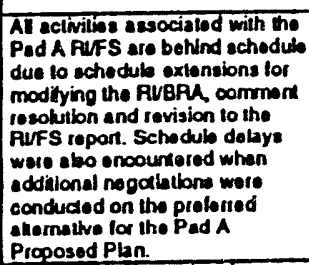 \\
\hline T.1.7.1.1.1.1 & 30 & 7.12 & Sion Final AOO & Bolore TN & $3 / 22 / 03$ & 1.1 .7 .1 .1 .1 .1 & 3 & 1.1.7.2.012 & 45 & $\bar{B}$ & 4 & 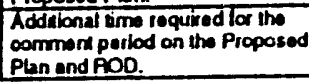 \\
\hline
\end{tabular}

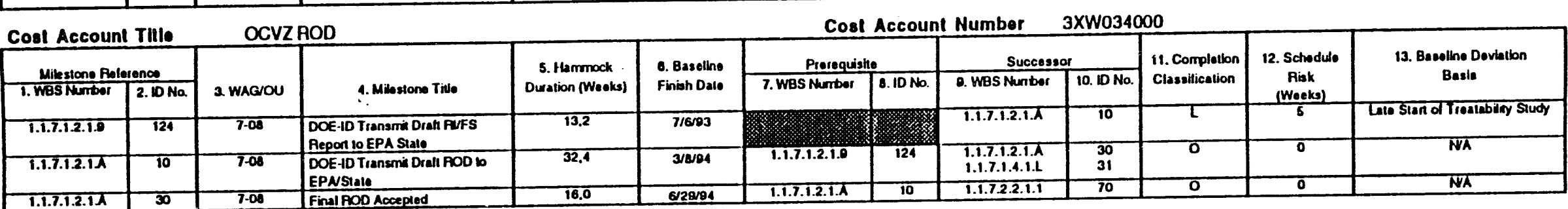

\section{Cost Account THI0 OCVZ Cleanup $\quad$ Cost Account Number $3 \times W C 34000$}

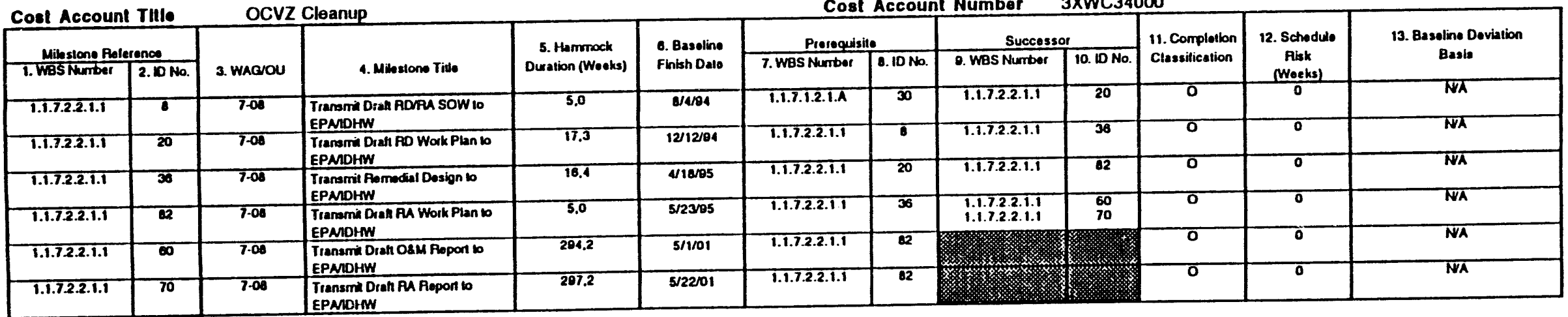

Cost Account Tlllo Pit 9 Comprehensive Demo

Cost Account Number $3 \times W 037000$

\begin{tabular}{|c|c|c|c|c|c|c|c|c|c|c|c|c|}
\hline \multicolumn{2}{|c|}{ Cost Account THle } & \multicolumn{2}{|c|}{ Pit 9 Comprehensive Demo } & & & Cost & Accour & Number & $X W 03 / 0$ & & \multirow{3}{*}{$\begin{array}{l}\text { 12. Schedule } \\
\text { Pisk } \\
\text { (Woeks) }\end{array}$} & \multirow{3}{*}{$\begin{array}{l}\text { 13. Baseoline Doviation } \\
\text { Basis }\end{array}$} \\
\hline Therente & 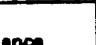 & \multirow[b]{2}{*}{ 3. WaGrou } & \multirow[b]{2}{*}{ 4. Mllosione Titie } & \multirow{2}{*}{$\begin{array}{c}\text { 5. Ilammock } \\
\text { Duration (Woeks) }\end{array}$} & \multirow{2}{*}{$\begin{array}{l}\text { 6. Baseline } \\
\text { Finish Dalo }\end{array}$} & \multicolumn{2}{|c|}{ Prorequisite } & \multicolumn{2}{|c|}{ Successor } & \multirow{2}{*}{$\begin{array}{l}\text { 11. Complotion } \\
\text { Clessillcation }\end{array}$} & & \\
\hline 1. Was Numbor & $2.10 \mathrm{No}$ & & & & & 7. WBS Nurber & $0.10 \mathrm{No}$. & D. WAS Nurtber & 10. $10 \mathrm{No}$ & & & \\
\hline 1.1 .7 .1 .3 .2 .4 & 68 & 7.10 & Ston $\mathrm{AOO}$ & 0.0 & $3 / 30193$ & & & 1.1 .7 .1 .3 .2 .4 & 23 & $\mathbf{L}$ & $\frac{100}{15}$ & 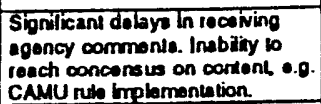 \\
\hline 1.1.7.1.3.2.4 & 23 & $7-10$ & $\begin{array}{l}\text { Drah Forru sow Submilod lor } \\
\text { Aovbow }\end{array}$ & 2.4 & 4/20/93 & 1.1 .7 .1 .3 .2 .4 & 58 & 1.1 .7 .1 .3 .2 .6 & 2 & 0 & 0 & NA \\
\hline 1.1.7.1.3.2.8 & 2 & $7 \cdot 10$ & $\begin{array}{l}\text { Aoview } \\
\text { EG\&G \& DOE-1D Approvi LPT } \\
\text { Aomodiation Option d } \\
\text { Subcontinct }\end{array}$ & 105.1 & $6 / 2 / 85$ & 1.1 .7 .1 .3 .2 .4 & 23 & 1.1.7.1.3.1.7 & 7 & $\mathbf{T}$ & 83 & $\begin{array}{l}\text { Rovision d schodule lo allow lime } \\
\text { lor lecility doslon and completion } \\
\text { of LPT. }\end{array}$ \\
\hline
\end{tabular}




\section{Schedule Contingency Worksheet}

Waste Area Group 7

Page 2 ol 3

\begin{tabular}{|c|c|c|c|c|c|c|c|c|c|c|c|c|}
\hline \multirow{2}{*}{\multicolumn{2}{|c|}{ Cost Account Tilie }} & \multicolumn{4}{|c|}{ TRU Contaminaled Pils \& Trenches } & \multicolumn{6}{|c|}{ Cost Account Number $3 \times w 035000$} & \multirow{3}{*}{$\begin{array}{l}\text { 13. Basoline Doviation } \\
\text { Besta }\end{array}$} \\
\hline & & \multirow[b]{2}{*}{ 3. WAQJOU } & \multirow[b]{2}{*}{ 4. Mibstono Tille } & \multirow{2}{*}{$\begin{array}{l}\text { 5. Hammock } \\
\text { Duration (Woeks) }\end{array}$} & \multirow{2}{*}{$\begin{array}{l}\text { O. Baseline } \\
\text { Finish Dale }\end{array}$} & \multicolumn{2}{|c|}{ Preceguisile } & \multicolumn{2}{|c|}{ Successor } & \multirow{2}{*}{$\begin{array}{l}\text { 11. Complotion } \\
\text { Classidication }\end{array}$} & \multirow{2}{*}{$\begin{array}{l}\text { 12. Schodulo } \\
\text { Pisk } \\
\text { Weeks) }\end{array}$} & \\
\hline T.WBS Nution & $2.10 \mathrm{No}$ & & & & & 7. WeS Number & $0.10 \mathrm{No}$ & O. WBS Nurtbor & $10.10 \mathrm{No}$ & & & \\
\hline 1.1.7.1.3.1.4 & $\pi$ & $7 \cdot 13$ & $\begin{array}{l}\text { Submi Drah SOW lor } \\
\text { EPMDHWDOE-HO Raview }\end{array}$ & 53,4 & 4/20/94 & & & 1.1.7.1.3.1.4 & 10 & $\tau$ & 20 & $\begin{array}{l}\text { Wow risk assossmorit strateog lo } \\
\text { moro complox than plamod. }\end{array}$ \\
\hline 1.1.7.1.3.1.4 & 10 & 7.13 & $\begin{array}{l}\text { Subrrit Draal RUFS Work Plan lor } \\
\text { Roviow lo EPMDHWID }\end{array}$ & 21.2 & \$/30/84 & 1.1.7.1.3.1.4 & 11 & 1.1 .7 .1 .3 .1 .7 & 7 & 0 & D & NA \\
\hline 1.1.7.1.3.1.7 & 7 & $7 \cdot 13$ & $\begin{array}{l}\text { Subrit Drath PIVS Report bo } \\
\text { EPNSiato }\end{array}$ & 81,3 & $5 / 20 / 98$ & $\begin{array}{l}T .1 .7 .1 .3 .1 .4 \\
1.1 .7 .1 .3 .2 .0 \\
1.1 .7 .1 .3 .1 .1\end{array}$ & $\begin{array}{l}10 \\
2 \\
22\end{array}$ & 1.1.7.1.3.1.8 & 10 & O & O & TWA \\
\hline ד.1.7.1.3.1. & 10 & $7 \cdot 13$ & $\begin{array}{l}\text { Submil Drah } \\
\text { ROORRosponsanoreses Surmary } \\
\text { LoEPMNDtiW }\end{array}$ & 41.3 & 4/1/87 & $\frac{1.1 .7 .1 .3 .1 .7}{1.1 .7}$ & $\frac{22}{7}$ & $\begin{array}{l}1.1 .7 .1 .3 .1 .8 \\
1.1 .1 .1 .4 .1 .1\end{array}$ & 3 & $\overline{0}$ & $\overline{0}$ & WA \\
\hline 1.1.7.1.3.1. & 0 & 7.13 & Final POD Aocepled & 77.1 & $0 / 1 / 197$ & 1.1 .7 .1 .3 .1 .8 & 10 & 1.1.7.2.013 & 150 & 0 & 0 & WA \\
\hline 1.1.7.1.3.11 & 10 & 7.13 & $\begin{array}{l}\text { Dran iA SOW Scomined to } \\
\text { EPMDHW }\end{array}$ & 53,4 & 4/20/84 & & & 1.1.7.1.3.1.A & 41 & $\bar{B}$ & 8 & $\begin{array}{l}\text { Now risk assosemmert ethategy is } \\
\text { moro comphex than ple nnod. }\end{array}$ \\
\hline 1.1.7.1.3.1K & 41 & $7 \cdot 13$ & $\begin{array}{l}\text { EPMUDHW } \\
\text { Transmi Drat } 4 \text { FOD } 10 \\
\text { EPMDHAW }\end{array}$ & 10.4 & 4/25/95 & $1.1 .7 .1 .3 .1 . A$ & 40 & 1.1.7.1.3.1. $\mathrm{K}$ & 22 & O & 0 & NA \\
\hline 1.1.7.1.3.1A & 22 & 7.13 & IA AOO Accepled & 16.0 & M17/85 & 1.1.7.1.3.1.A & 44 & 1.1.7.2.13 & 450 & o & 0 & $\mathrm{~W} / \mathrm{K}$ \\
\hline
\end{tabular}

\begin{tabular}{|c|c|c|c|c|c|c|c|c|c|c|c|c|}
\hline \multicolumn{2}{|c|}{ Cost Account Tillo } & \multicolumn{2}{|c|}{ WAG 7 Comprehensive ROD } & \multicolumn{9}{|c|}{ Cost Account Number $3 \times W 041000$} \\
\hline Milestone Piel & lenos & \multirow[b]{2}{*}{ 3. WAGNOU } & \multirow[b]{2}{*}{ 4. Mibstome Tallo } & \multirow{2}{*}{$\begin{array}{l}\text { 5. Hamrnock } \\
\text { Duation (Wooks) }\end{array}$} & \multirow{2}{*}{$\begin{array}{l}\text { C. Baselino } \\
\text { Finish Dasto }\end{array}$} & \multicolumn{2}{|c|}{ Prerequisine } & \multicolumn{2}{|c|}{ Successor } & \multirow{2}{*}{$\begin{array}{l}\text { 11. Complotion } \\
\text { Claseatication }\end{array}$} & \multirow{2}{*}{$\begin{array}{l}\text { 12. Schodulo } \\
\text { Pink } \\
\text { (Weeks) }\end{array}$} & \multirow{2}{*}{$\begin{array}{l}\text { 93. Beseline Doviation } \\
\text { Basis }\end{array}$} \\
\hline 1. Was Nurrtar & $2.10 \mathrm{No}$ & & & & & 7. WaS Number & $8.10 \mathrm{No}$ & 9. WBS Numbor & 10. $10 \mathrm{No}$. & & & \\
\hline 1.1.7.1.4.8.E & 10 & 7.03 & $\begin{array}{l}\text { Dran SOW Submined } 10 \\
\text { EPAMDHW }\end{array}$ & 115.2 & $7 / 28 / 85$ & & & 1.1.7.1.4.1.E & 44 & 0 & 0 & NA \\
\hline 1.1.7.1.4.1.E & 14 & 7.03 & Irangmi Dralf ROD WEPMMDHW & 48.4 & $7 / 10 / 98$ & 1.1.7.1.1.1.E & 10 & 1.1.7.1.1.1.E & 22 & 0 & 0 & NA \\
\hline 1.1.7.1.4.1.E & 22 & 7.03 & Finel FOO Aocopted & 10.0 & $11 / 12 / 96$ & 1.1.7.1.4.1.E & 14 & $\begin{array}{l}7.1 .7 .1 .3 .1 .7 \\
1.1 .7 .2 .003\end{array}$ & $\begin{array}{l}7 \\
450 \\
\end{array}$ & б & 0 & NA \\
\hline 1.1.7.1.4.2. & 33 & 7.00 & Dellver Final Intormal Ropon & 123.1 & \$20/95 & & & 1.1.7.1.4.1.K & 8 & o & 0 & NA \\
\hline 1.1.7.1.4.1.K & T & $7-14$ & $\begin{array}{l}\text { Scomi SOW } 10 \text { DOE-TD lor } \\
\text { Lanaminal lo EPMDAHY }\end{array}$ & 5.2 & 10227105 & 1.1.7.1.4.2. B & 33 & T.1.7.1.1.1.K & 34 & 0 & $\overline{0}$ & TW \\
\hline 8.1.7.1.4.1.K & 34 & 7.14 & $\begin{array}{l}\text { Submi WAG } 7 \text { Comp. Dath RUFS } \\
\text { Wack Plan lo EPMDOHW }\end{array}$ & 20.1 & $4 / 1 / 90$ & 1.1.7.1.4.1.K & 8 & 1.1.7.1.4.1.K & 31 & 0 & o & NA \\
\hline $1.1 .7 .1 .4 .1 . \mathrm{L}$ & 31 & $7-14$ & $\begin{array}{l}\text { SLbmid WAG TComp. Dnit RAFS } \\
\text { Repont to EPMDHW }\end{array}$ & 82.4 & $11 / 25 / 67$ & $\begin{array}{l}1.1 .7 .1 .1 . \mathrm{K} \\
1.1 .7 .1 .1 .1 .1 \\
1.1 .1 .1 .1 . \mathrm{A} \\
1.1 .1 .1 .1 .8\end{array}$ & $\begin{array}{l}4 \\
3 \\
10 \\
10 \\
\end{array}$ & $\begin{array}{l}1.1 .10 .1 .1 .1 .2 \\
1.1 .7 .1 .4 .1 .6\end{array}$ & $\begin{array}{l}30 \\
13\end{array}$ & 0 & 0 & $\overline{N A}$ \\
\hline 1.1.7.1.1.1. C & 13 & 7.14 & SLbmi Drath POD 10 EPMDHW & 63.3 & $3 / 1609$ & 1.1.7.1.1.1. & 31 & 1.1.7.1.1.1.C & 30 & 0 & 0 & $\mathbf{N A}$ \\
\hline $1.1 .7 .1 .4 .1 . \mathrm{C}$ & 30 & 7.14 & Final ROO Aoceplod & 20.2 & $8 / 9180$ & 1.1.7.1.1.1.C & 13 & & 8 & 0 & 0 & NA \\
\hline
\end{tabular}

\begin{tabular}{|c|c|c|c|c|c|c|c|c|c|c|c|c|}
\hline \multirow{3}{*}{\multicolumn{2}{|c|}{ Cost Account THlo }} & \multirow{2}{*}{\multicolumn{4}{|c|}{ OU 7-03 RWMC Non-TRU Pils \& Trenches }} & \multicolumn{4}{|c|}{ Cost Account Number $\quad 1.1 .7 .2 .03$} & \multirow{4}{*}{$\begin{array}{l}\text { 11. Completion } \\
\text { Classidication }\end{array}$} & \multirow{4}{*}{$\begin{array}{l}\text { 12. Schadule } \\
\text { Aisk } \\
\text { (Weeks) }\end{array}$} & \multirow{4}{*}{$\begin{array}{l}\text { 13. Basellno Deviation } \\
\text { Basis }\end{array}$} \\
\hline & & & & & & \multirow{2}{*}{\multicolumn{2}{|c|}{ Preloquisile }} & \multirow{2}{*}{\multicolumn{2}{|c|}{ Succossor }} & & & \\
\hline & & \multirow[b]{2}{*}{ 3. WhG } & \multirow[b]{2}{*}{ 4. Mibstione Tille } & \multirow{2}{*}{$\begin{array}{l}\text { S. Hommock } \\
\text { Duration (Woeks) }\end{array}$} & \multirow{2}{*}{$\begin{array}{l}\text { 6. Baseline } \\
\text { Finish Dale }\end{array}$} & & & & & & & \\
\hline 1. Was Number & 2. $10 \mathrm{Na}$ & & & & & 7. WBS Nurtiber & D. $10 \mathrm{NO}$. & O. WBS Number & $10.10 \mathrm{No}$. & & & \\
\hline 1.1 .7 .2 .003 & 450 & $7-03$ & Submi Drah SOW/AD Work Plan & 13,0 & 6/22/05 & 1.1.7.1.4.1.E & 22 & $\begin{array}{l}1.1 .7 .2 .003 \\
1.1 .7 .2 .003\end{array}$ & $\begin{array}{l}100 \\
213\end{array}$ & 0 & 0 & NA \\
\hline 1.1.7.2.003 & 100 & 7.03 & Subrit Drah Design lo EPMDLWW & 10.0 & $11 / 06 / 95$ & $\begin{array}{l}1.1 .7 .2 .003 \\
1.1 .7 .2 .003\end{array}$ & $\begin{array}{l}450 \\
213\end{array}$ & $\begin{array}{l}T .1 .7 .2 .003 \\
1.1 .7 .2 .003\end{array}$ & $\begin{array}{l}617 \\
017\end{array}$ & 0 & 0 & NA \\
\hline 1.1.7.2.003 & $\overline{213}$ & $7-03$ & $\begin{array}{l}\text { Submit Dran fu Work Plen to } \\
\text { EPMDIWW }\end{array}$ & 10.0 & $11 / 06 / 85$ & 1.1.7.2.003 & 450 & $\begin{array}{l}1.1 .7 .2 .003 \\
1.1 .7 .2 .003 \\
1.1 .1 .2 .003 \\
\end{array}$ & $\begin{array}{l}100 \\
017 \\
017\end{array}$ & 0 & 0 & TWA \\
\hline T.1.7.2.003 & 617 & 7.03 & $\begin{array}{l}\text { Submit Dian FA Repont to } \\
\text { EPMDHW }\end{array}$ & 201,3 & $12 / 02 / 89$ & $\begin{array}{l}1.1 .7 .2 .003 \\
1.1 .7 .2 .003\end{array}$ & $\begin{array}{l}108 \\
213\end{array}$ & 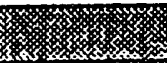 & & 0 & $\overline{0}$ & NA \\
\hline 1.1.7.2.003 & 917 & $7-03$ & $\begin{array}{l}\text { Submi Drath O\&M Roport io } \\
\text { EPMOrW }\end{array}$ & 205.0 & $1 / 04 / 00$ & $\begin{array}{l}1.1 .7 .2 .003 \\
1.1 .7 .2 .003 \\
\end{array}$ & $\begin{array}{l}708 \\
213 \\
\end{array}$ & & & 0 & $\overline{0}$ & $\overline{\text { NA }}$ \\
\hline
\end{tabular}


Schedule Contingency Worksheet Waste Area Group 7

Page 3 of 3

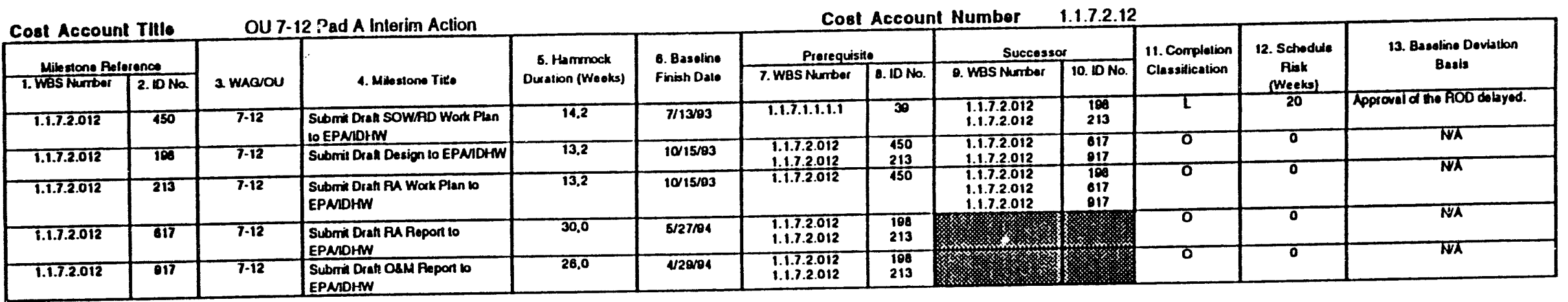

Cost Account TIlle OU 7-13 TRU Contaminaled Waste Pits \& Trenclies

\begin{tabular}{|c|c|c|c|c|c|}
\hline \multicolumn{2}{|c|}{ Mingsione Polerence } & \multirow[b]{2}{*}{ 3. WhaNoU } & \multirow[b]{2}{*}{ 4. Mibstorn Tillo } & \multirow{2}{*}{$\begin{array}{l}\text { 5. Hammock } \\
\text { Duration (Weoks) }\end{array}$} & \multirow{2}{*}{$\begin{array}{l}\text { O. Basoline } \\
\text { Frobsh Date }\end{array}$} \\
\hline T. WoS Number & $2.10 \mathrm{No}$ & & & & \\
\hline 1.1.7.2.013 & 450 & $7 \cdot 13$ & $\begin{array}{l}\text { SLbmin Drall SOW/AD Work Plan } \\
\text { lo EPMIDIW }\end{array}$ & 13.0 & $\$ / 2390$ \\
\hline 1.1.7.2.013 & 190 & $7 \cdot 13$ & Sutmil Drat Dosign to EPMDHWW & 18.0 & $2 / 00 / 90$ \\
\hline 1.1.7.2.013 & 213 & 7.13 & $\begin{array}{l}\text { Subrim Drall Fu Work Plan io } \\
\text { EPNDOHW }\end{array}$ & 18.0 & $2 / 00 / 90$ \\
\hline 1.1.7.2.013 & 617 & 7.13 & $\begin{array}{l}\text { Submi Drath Pu fiepont to } \\
\text { EPMOHW }\end{array}$ & 37.1 & $11 / 02 / 09$ \\
\hline T.1.7.2.013 & 017 & $7-13$ & $\begin{array}{l}\text { Submin Dian OSM Ropon lo } \\
\text { EPADHHW }\end{array}$ & 41.1 & $12 / 03 / 89$ \\
\hline
\end{tabular}

Cost Account Number $\quad$ 1.1.7.2.13

\begin{tabular}{|c|c|c|c|c|c|c|}
\hline \multicolumn{4}{|c|}{ Cost Account Number } & \multirow{3}{*}{$\begin{array}{l}\text { 11. Compleation } \\
\text { Clasesflication }\end{array}$} & \multirow{3}{*}{$\begin{array}{l}\text { 12. Schodulo } \\
\text { Ptok } \\
\text { (Wooke) }\end{array}$} & \multirow{3}{*}{$\begin{array}{l}\text { 13. Bareline Doviation } \\
\text { Beats }\end{array}$} \\
\hline \multicolumn{2}{|c|}{ Prorequisite } & \multicolumn{2}{|c|}{ Successor } & & & \\
\hline 7. WBS Number & $0.10 \mathrm{No}$. & 0. WBS Numbor & 10.10 No. & & & \\
\hline 1.1 .7 .1 .3 .1 .8 & 0 & $\begin{array}{l}1.1 .7 .2 .013 \\
\text { i.1.7.2.013 }\end{array}$ & $\begin{array}{l}109 \\
213\end{array}$ & 0 & 0 & NA \\
\hline $\begin{array}{l}1.1 .7 .2 .013 \\
1.1 .7 .2 .013\end{array}$ & $\begin{array}{l}450 \\
213\end{array}$ & 1.1 .7 .2 .013 & 817 & 0 & $\overline{0}$ & NA \\
\hline$\frac{1.1 .7 .2 .013}{3}$ & 150 & $\begin{array}{l}11.7 .2 .013 \\
11.7 .2 .013 \\
1.1 .7 .2 .013\end{array}$ & $\begin{array}{l}110 \\
1017 \\
017\end{array}$ & 0 & $\overline{0}$ & NA \\
\hline $\begin{array}{l}1.1 .7 .2 .013 \\
1.1 .7 .2 .013\end{array}$ & $\begin{array}{l}108 \\
213\end{array}$ & & & 0 & $\overline{0}$ & NA \\
\hline $\begin{array}{l}1.1 .7 .2 .013 \\
1.1 .7 .2 .013\end{array}$ & $\begin{array}{l}708 \\
213\end{array}$ & & 缀 & 0 & 0 & NA \\
\hline
\end{tabular}

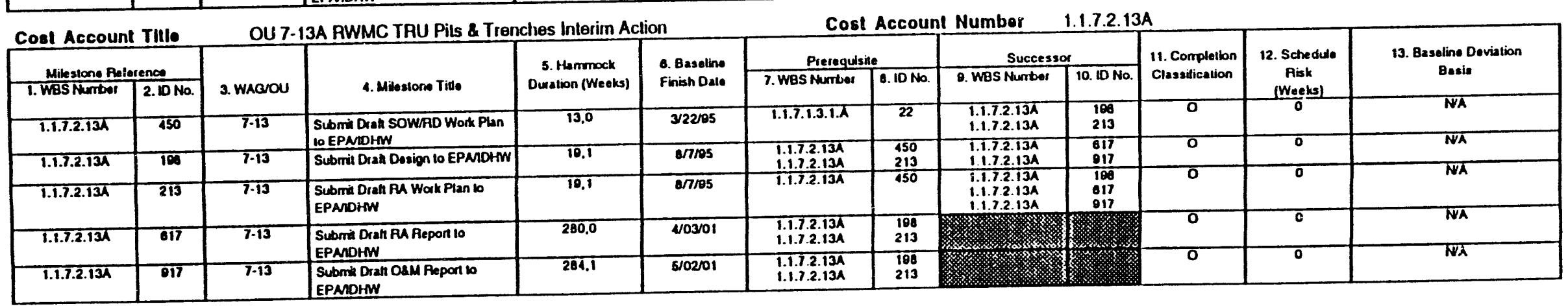


Schedule Contingency Worksheet

Waste Area Group 10

Page 1 of 1

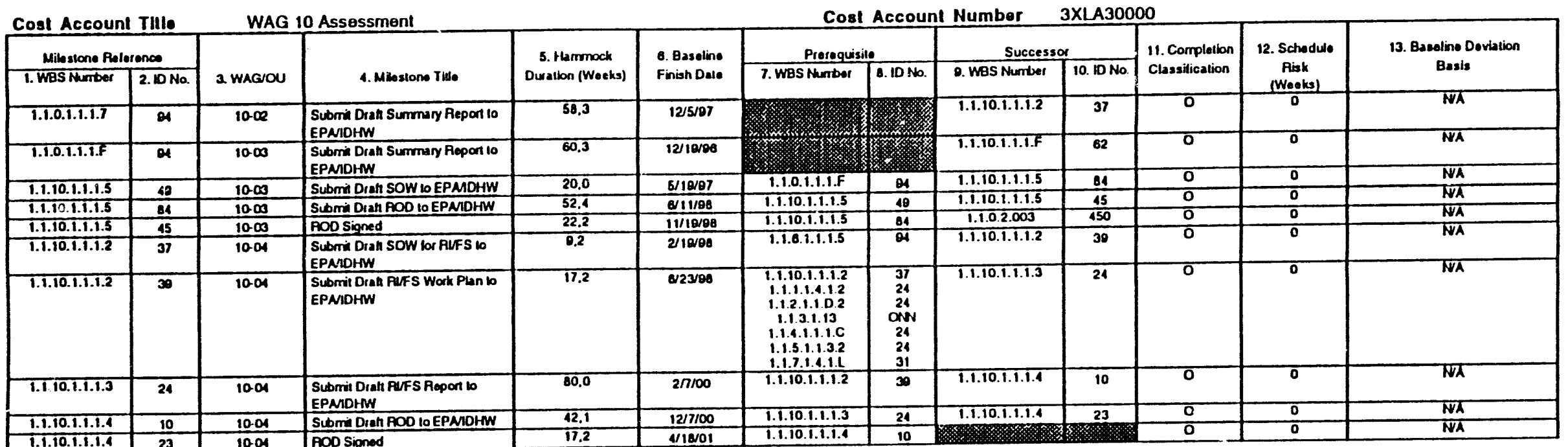

Cost Account THtle WAG 10-03 Ordnance Removal and Remediation

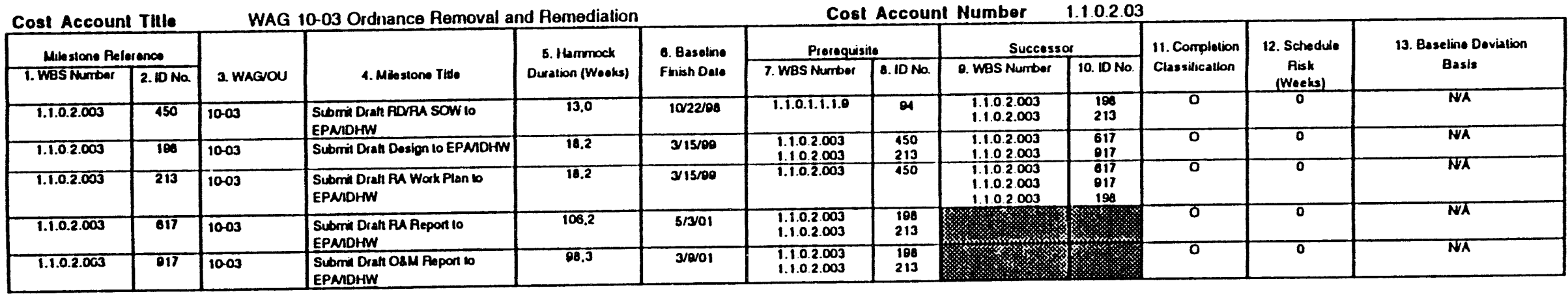

Cost Account Tllle $\quad 10-05$ Ordnance Interim Aclion

\begin{tabular}{|c|c|c|c|c|c|c|c|c|c|c|c|c|}
\hline \multirow{2}{*}{\multicolumn{2}{|c|}{$\frac{\text { Cost Account Tllle }}{\text { Milostono Paterenos }}$}} & \multicolumn{2}{|c|}{ 10-05 Ordnance Interim Action } & \multicolumn{6}{|c|}{ Cost Account Number } & \multirow{3}{*}{$\begin{array}{l}\text { 11. Conplotion } \\
\text { Clessitication }\end{array}$} & \multirow{3}{*}{$\begin{array}{l}\text { 12. Scthodulo } \\
\text { Risk } \\
\text { (Weeks) }\end{array}$} & \multirow{3}{*}{$\begin{array}{l}\text { 13. Baseline Deviation } \\
\text { Besis }\end{array}$} \\
\hline & & \multirow[b]{2}{*}{ 3. WAGNOU } & \multirow[b]{2}{*}{ 4. Minstome Tillo } & \multirow{2}{*}{$\begin{array}{l}\text { 5. Harmmock } \\
\text { Duwation (Woeks) }\end{array}$} & \multirow{2}{*}{$\begin{array}{l}\text { O. Basoline } \\
\text { Finish Dale }\end{array}$} & \multicolumn{2}{|c|}{ Prerequisile } & \multicolumn{2}{|c|}{ Succossor } & & & \\
\hline 1. WaS Nuntber & 2.10 No. & & & & & 7. WBS Nurrbot & $0.10 \mathrm{No}$. & 9. WeS iturtitor & 10. $10 \mathrm{No}$. & & & \\
\hline 1.1.0.2.005 & 817 & 10.05 & $\begin{array}{l}\text { Submi Drah FM Repon to } \\
\text { EPMIDHW }\end{array}$ & 48.3 & $3 / 10 / 84$ & & & & & 0 & 0 & NA \\
\hline
\end{tabular}

Cost Account TIIlo WAG 10 Rad Conlaminaled Soils

Cost Account Number $3 \times 1810000$

\begin{tabular}{|c|c|c|c|c|c|c|c|c|c|c|c|c|}
\hline \multicolumn{2}{|c|}{ Cost Account TIIle } & \multicolumn{2}{|c|}{ WAG 10 Pad Conlaminaled Soils } & \multicolumn{9}{|c|}{ Cost Account Number $3 \times 1410000$} \\
\hline Mile stone fie & ence & \multirow[b]{2}{*}{ 3. WAGUOU } & \multirow[b]{2}{*}{ 4. Mibstone Tillo } & \multirow{2}{*}{$\begin{array}{c}\text { S. Hammock } \\
\text { Duration (Woeks) }\end{array}$} & \multirow{2}{*}{$\begin{array}{l}\text { 6. Baselino } \\
\text { Finish Date }\end{array}$} & \multicolumn{2}{|c|}{ Prelequizile } & \multicolumn{2}{|c|}{ Successor } & \multirow{2}{*}{$\begin{array}{l}\text { 11. Completion } \\
\text { Classilication }\end{array}$} & \multirow{2}{*}{$\begin{array}{l}\text { 12. Schodulo } \\
\text { Risk } \\
\text { (Weaks) }\end{array}$} & \multirow{2}{*}{$\begin{array}{l}\text { 13. Baseline Dovialion } \\
\text { Basla }\end{array}$} \\
\hline 1. WoS sumber & 2.10 No. & & & & & 7. WBS Nurtbor & $0.10 \mathrm{No}$ & O. WaS Number & 10. $10 \mathrm{No}$. & & & \\
\hline 1.1 .10 .1 .1 .4 .1 & $\boldsymbol{\infty}$ & & Submi Diall SOW IO EPMDHWW & 2.1 & $4 / 15 / 93$ & & & & 䋞 & $T$ & 10 & 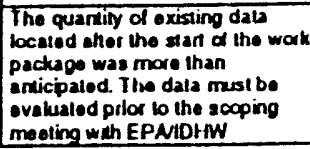 \\
\hline
\end{tabular}


APPENDIX C

\section{GANTT CHART SCHEDULES}




\section{BASELINE SCHEDULE RISK}

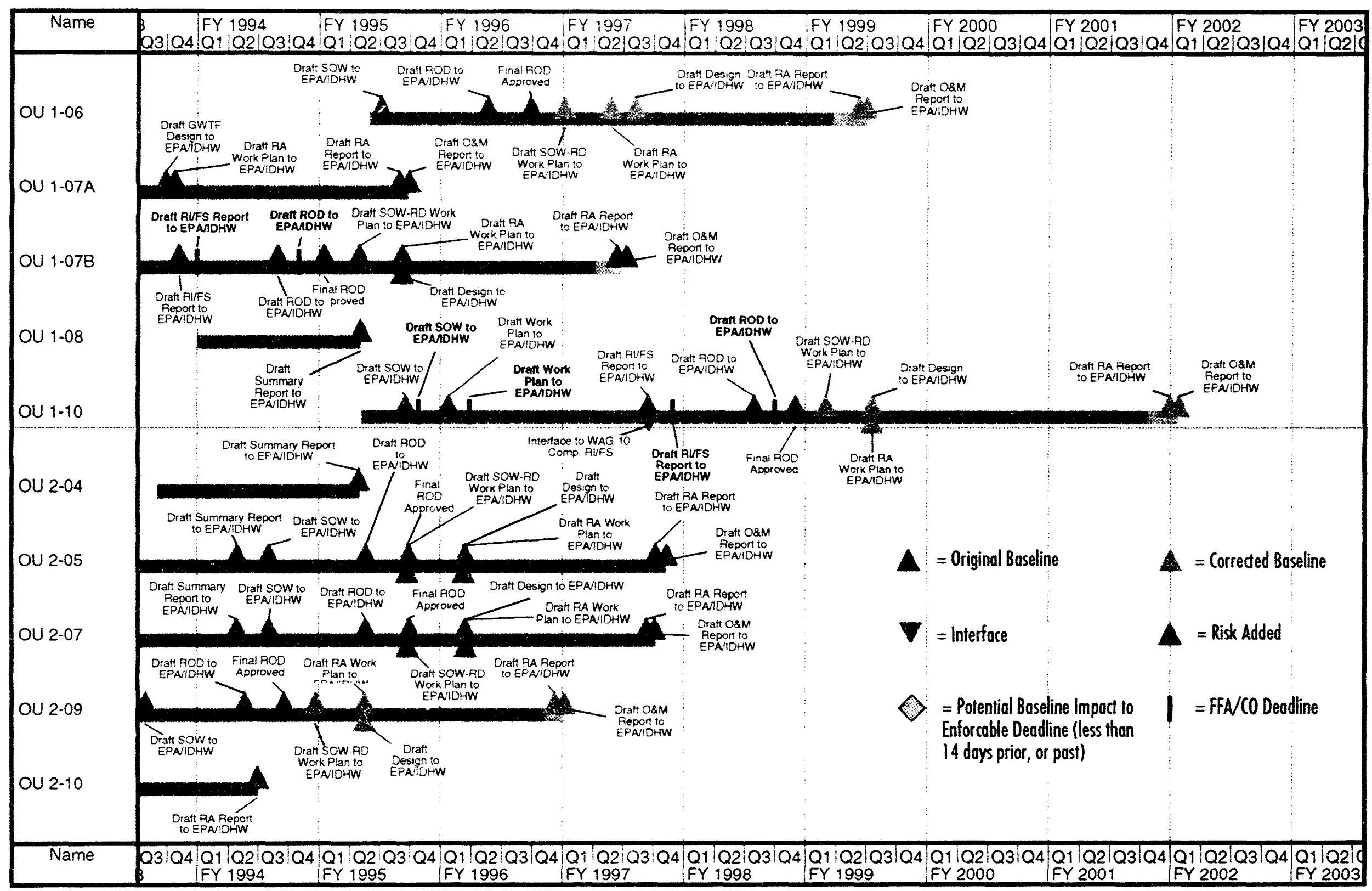




\section{BASELINE SCHEDULE RISK}

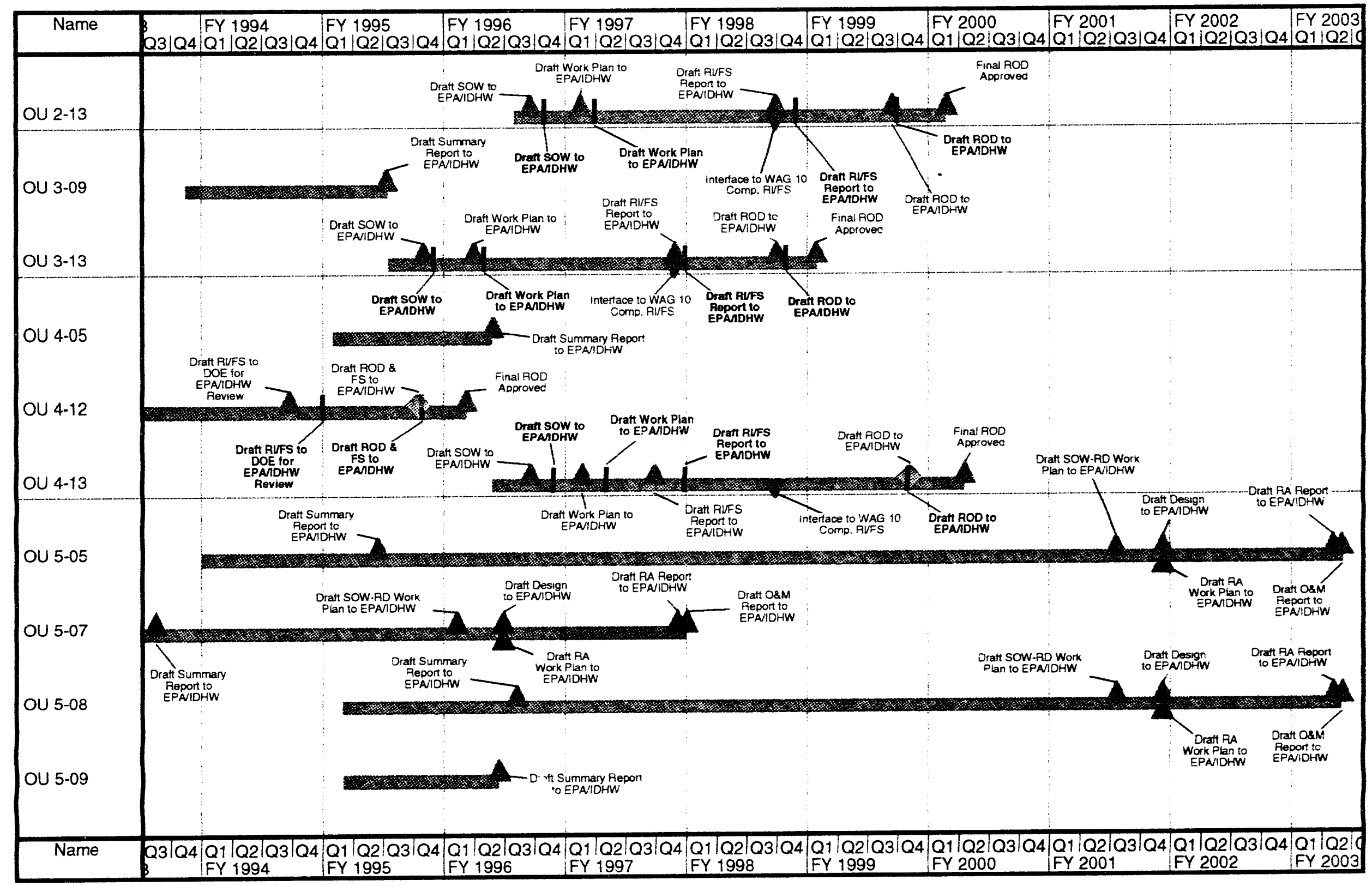




\section{BASELINE SCHEDULE RISK}

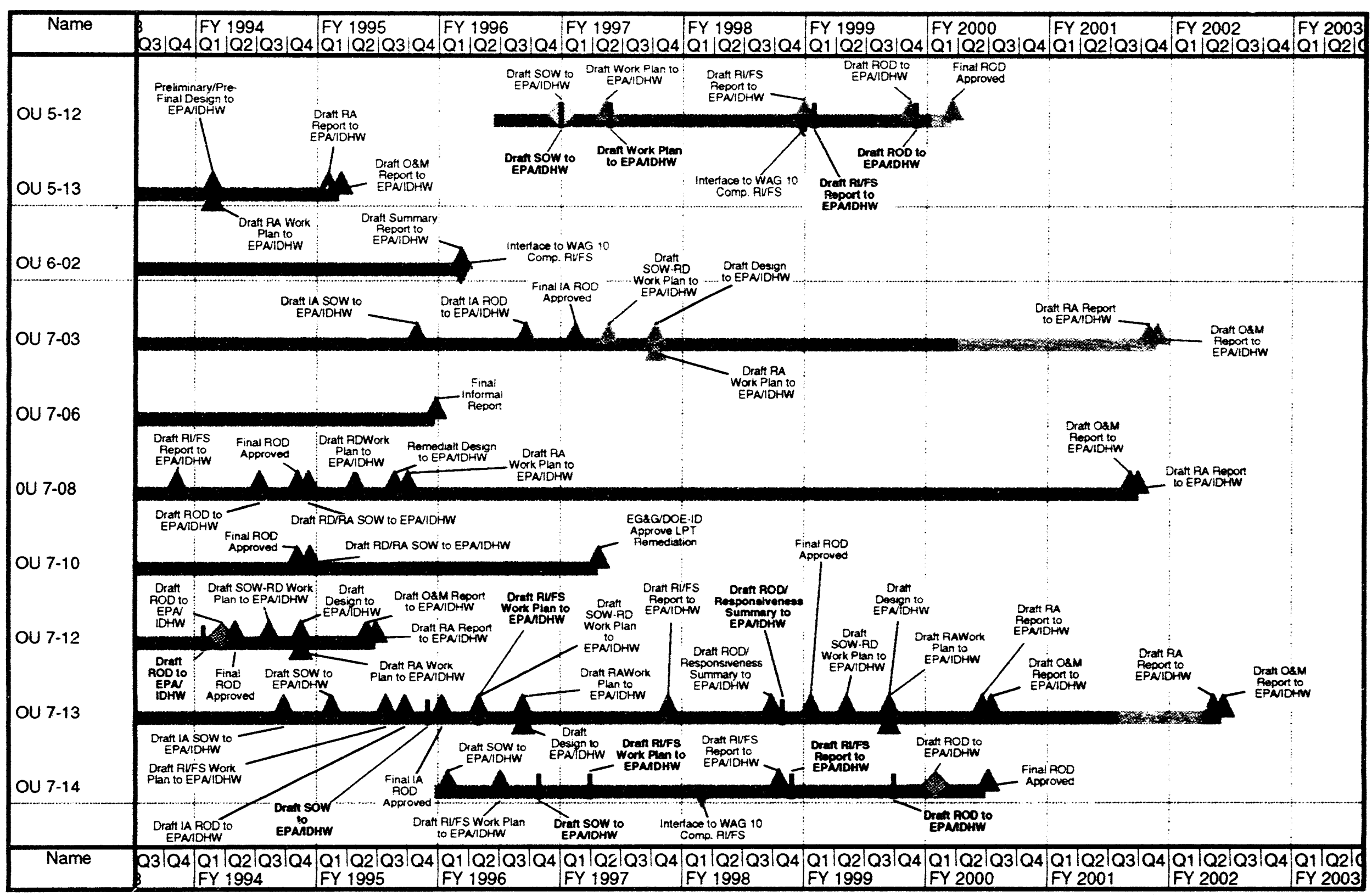




\section{BASELINE SCHEDULE RISK}

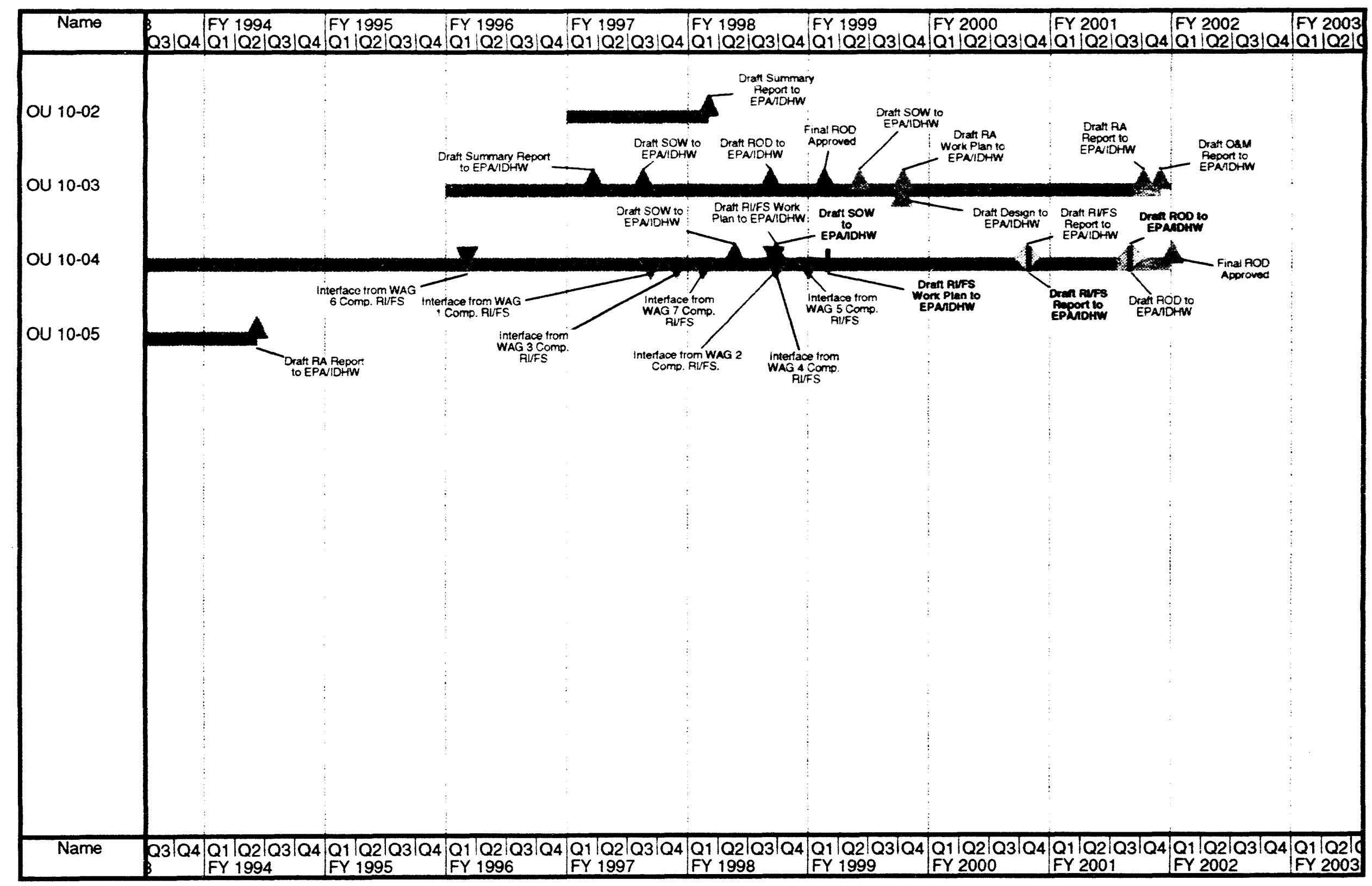


APPENDIX D

DATA TABLES 


\begin{tabular}{llll}
\hline OU 1-06 & Draft SOW to EPAIDHW & $\mathbf{0 4 - 0 4 - 9 5}$ & \\
& Draft ROD to EPAIDHW & $\mathbf{0 2 - 2 3 - 9 6}$ & \\
& Final ROD Approved & $\mathbf{0 6 - 2 7 - 9 6}$ & \\
& Draft SOW-RD Work Plan to EPAIDHW & $\mathbf{0 6 - 2 7 - 9 8}$ & $\mathbf{1 0 - 0 3 - 9 8}$ \\
& Draft Design to EPAIDHW & $\mathbf{1 1 - 0 7 - 9 6}$ & $\mathbf{0 2 - 2 5 - 9 7}$ \\
& Draft RA Work Plan to EPAIDHW & $\mathbf{1 1 - 0 7 - 9 6}$ & $\mathbf{0 2 - 2 5 - 9 7}$ \\
& Draft RA Report to EPAIDHW & $\mathbf{1 1 - 2 0 - 9 8}$ & $\mathbf{0 3 - 0 9 - 9 9}$ \\
& Draft O\&M Report to EPAIDHW & $\mathbf{1 2 - 2 2 - 9 8}$ & $\mathbf{0 4 - 0 6 - 9 9}$
\end{tabular}

\begin{tabular}{lll}
\hline OU 1-07A & Draft GWTF Design to EPAIDHW & $\mathbf{0 7 - 2 6 - 9 3}$ \\
& Draft RA Work Plan to EPAIDHW & $\mathbf{0 7 - 0 2 - 9 3}$ \\
& Draft RA Report to EPAIDHW & $\mathbf{0 5 - 3 1 - 9 5}$ \\
& Draft O\&M Report to EPAIDHW & $\mathbf{0 6 - 3 0 - 9 5}$
\end{tabular}

\begin{tabular}{|c|c|c|c|c|}
\hline $\mathrm{OU} 1-07 \mathrm{~B}$ & $\begin{array}{l}\text { Draft RI/FS Report to EPAIDHW } \\
\text { Draft ROD to EPAIDHW } \\
\text { Final ROD Approved } \\
\text { Draft SOW-RD Work Plan to EPAIDHW } \\
\text { Draft Design to EPAIDHW } \\
\text { Draft RA Work Plan to EPAIDHW } \\
\text { Draft RA Report to EPAIDHW } \\
\text { Draft O\&M Report to EPAIDHW }\end{array}$ & $\begin{array}{l}07-26-93 \\
05-17-94 \\
10-03-94 \\
10-24-94 \\
03-13-95 \\
03-13-95 \\
12-09-96 \\
01-10-97\end{array}$ & $\begin{array}{l}01-13-95 \\
05-22-95 \\
05-22-95 \\
02-26-97 \\
03-24-97\end{array}$ & $\begin{array}{l}08-09-93 \\
06-01-94 \\
10-17-94 \\
01-27-95 \\
06-06-95 \\
06-06-95 \\
03-12-97 \\
04-07-97\end{array}$ \\
\hline
\end{tabular}

\begin{tabular}{|c|c|c|c|c|}
\hline OU 1-08 & Draft Summary Report to EPAIDHW & $02-03-95$ & & \\
\hline OU 1-10 & $\begin{array}{l}\text { Draft SOW to EPAIDHW } \\
\text { Draft Work Plan to EPAIDHW } \\
\text { Draft RI/FS Report to EPAIDHW } \\
\text { Draft ROD to EPAIDHW } \\
\text { Final ROD Approved } \\
\text { Draft SOW-RD Work Plan to EPAIDHW } \\
\text { Draft Design to EPAIDHW } \\
\text { Draft RA Work Plan to EPAIDHW } \\
\text { Draft RA Report to EPAIDHW } \\
\text { Draft O\&M Report to EPAIDHW }\end{array}$ & $\begin{array}{l}06-16-95 \\
10-23-95 \\
06-17-97 \\
04-30-98 \\
08-31-98 \\
08-31-98 \\
01-18-99 \\
01-18-99 \\
06-25-01 \\
07-24-01\end{array}$ & $\begin{array}{l}12-04-98 \\
04-20-99 \\
04-20-99 \\
09-26-01 \\
10-24-01\end{array}$ & $\begin{array}{l}07-31-95 \\
12-31-95 \\
08-31-97 \\
08-30-98\end{array}$ \\
\hline OU 2.04 & Draft Summary Report to EPAIDHW & $01-31-95$ & & \\
\hline OU 2-05 & $\begin{array}{l}\text { Draft Summary Report to EPAIDHW } \\
\text { Draft SOW to EPAIDHW } \\
\text { Draft ROD to EPAIDHW } \\
\text { Final ROD Approved } \\
\text { Draft SOW-RD Work Plan to EPAIDHW } \\
\text { Draft Design to EPAIDHW } \\
\text { Draft RA Work Plan to EPAIDHW } \\
\text { Draft RA Report to EPAIDHW } \\
\text { Draft O\&M Report to EPAIDHW }\end{array}$ & $\begin{array}{l}01-31-94 \\
05-02-94 \\
02-20-95 \\
06-22-95 \\
07-25-95 \\
12-11-95 \\
12-11-95 \\
07-10-97 \\
08-08-97\end{array}$ & & \\
\hline OU 2-07 & $\begin{array}{l}\text { Draft Summary Report to EPAIDHW } \\
\text { Draft SOW to EPAIDHW }\end{array}$ & $\begin{array}{l}01-31-94 \\
05-02-94\end{array}$ & & \\
\hline
\end{tabular}


Draft ROD to EPAIDHW

Finai ROD Approved

Draft SOW-RD Work Plan to EPAIDHW

Draft Design to EPAIDHW

Draft RA Work Plan to EPAIDHW

Draft RA Report to EPAIDHW

Draft O\&M Report to EPAIDHW
02-20-95
06-22-95
07-25-95
12-11-95
12-11-95
06-09-97
07-09-97

04-30-93

02-18-94

06-22-94

07-26-94

12-12-94

12-12-94

07-10-96

08-08-96
09-23-94

02-17-95

02-17-95

09-09-96

10-08-96

\begin{tabular}{llll}
\hline OU 2-10 & Draft RA Report to EPAIDHW & $03-31-94$ & $07-31-96$ \\
\hline OU 2-13 & Draft SOW to EPAIDHW & $06-18-96$ & $12-31-96$ \\
& Draft Work Plan to EPAIDHW & $11-18-96$ & $08-31-98$ \\
& Draft RI/FS Report to EPAIDHW & $06-25-98$ & $06-30-99$ \\
& Draft ROD to EPAIDHW & $06-14-99$ & $11-22-99$ \\
& Final ROD Approved & & $04-14-95$ \\
\hline OU3-09 & Draft Summary Report to EPAIDHW & & $08-31-95$ \\
& & $08-01-95$ & $01-31-96$ \\
\hline OU 3-13 & Draft SOW to EPAIDHW & $01-01-96$ & $09-30-97$ \\
& Draft Work Plan to EPAIDHW & $09-01-97$ & $07-01-98$ \\
& Draft RI/FS Report to EPAIDHW & $10-30-98$ & $07-31-98$
\end{tabular}

\begin{tabular}{llll}
\hline OU 4-05 & Draft Summary Report to EPAIDHW & $02-23-96$ & $09-30-94$ \\
\hline OU 4-12 & Draft RI/FS Report to EPAIDHW & $06-23-94$ & $07-31-95$ \\
& $\begin{array}{l}\text { Draft ROD to EPAIDHW } \\
\text { Final ROD Approved }\end{array}$ & $12-08-95$ & \\
& & $06-18-96$ & $08-31-96$ \\
\hline OU 4-13 & Draft SOW to EPAIDHW & $11-18-96$ \\
& Draft Work Plan to EPAIDHW & $06-25-98$ & $01-31-97$ \\
& Draft RI/FS Report to EPAIDHW & $08-03-99$ & $07-31-99$ \\
& Draft ROD to EPAIDHW & $01-19-00$ & \\
\hline & Final ROD Approved & $03-15-95$ \\
\hline OU 5-05 & Draft Summary Report to EPAIDHW & $04-23-01$ \\
& Draft SOW-RD Work Plan to EPAIDHW & $09-07-01$ \\
& Draft Design to EPAIDHW & $09-07-01$
\end{tabular}


Draft RA Report to EPAIDHW

Draft O\&M Report to EPAIDHW
02-03-03

03-06-03

$\begin{array}{ll}06-16-93 & 05-18-93 \\ 10-24-94 & 11-09-95 \\ 03-13-95 & 03-28-96 \\ 03-13-95 & 03-28-96 \\ 08-19-96 & 09-05-97 \\ 09-17-96 & 10-03-97\end{array}$

\begin{tabular}{lll}
\hline OU 5-08 & Draft Summary Report to EPAIDHW & 05-09-96 \\
& Draft SOW-RD Work Plan to EPAIDHW & $\mathbf{0 4 - 2 3 - 0 1}$ \\
& Draft Design to EPAIDHW & $\mathbf{0 9 - 0 7 - 0 1}$ \\
& Draft RA Work Plan to EPAIDHW & $\mathbf{0 9 - 0 7 - 0 1}$ \\
& Draft RA Report to EPAVIDHW & $\mathbf{0 2 - 0 3 - 0 3}$ \\
& Draft O\&M Report to EPAIDHW & $\mathbf{0 3 - 0 6 - 0 3}$
\end{tabular}

OU 5-09 Draft Summary Report to EPAIIDHW 03-12-96

Draft Work Plan to EPAIDHW

Draft RI/FS Report to EPAIDHW

12-09-96

10-02-96

09-30-96

Draft ROD to EPAIDHW

07-30-98

02-13-97

02-28-97

Final ROD Approved

06-11-99

09-29-98

10-31-98

10-13-99 12-15-99

$08-31-99$

\begin{tabular}{llll}
\hline OU 5-13 & PreVPre-Final Design to EPAIIDHW & $\mathbf{0 7 - 2 3 - 9 3}$ & $\mathbf{1 1 - 2 3 - 9 3}$ \\
& Draft RA Work Plan to EPAIDHW & $\mathbf{0 8 - 0 9 - 9 3}$ & $\mathbf{1 1 - 2 3 - 9 3}$ \\
& Draft RA Report to EPAIDHW & $\mathbf{0 7 - 2 0 - 9 4}$ & $\mathbf{1 1 - 0 2 - 9 4}$ \\
& Draft O\&M Report to EPAIDHW & $\mathbf{0 8 - 2 2 - 9 4}$ & $\mathbf{1 2 - 0 8 - 9 4}$
\end{tabular}

\begin{tabular}{llll}
\hline OU 6-02 & Draft Summary Report to EPAIDHW & $12-07-95$ & \\
& & & \\
\hline OU 7-03 & Draft IA SOW to EPAIDHW & $07-26-95$ & \\
& Draft IA ROD to EPAIIDHW & $07-19-96$ & \\
& IA ROD Approved & $11-12-96$ & \\
& Draft SOW-RD Work Plan to EPAIDHW & $06-22-95$ & $\mathbf{0 2 - 2 4 - 9 7}$ \\
& Draft Design to EPAIDHW & $11-06-95$ & $07-09-97$ \\
& Draft RA Work Plan to EPAIDHW & $\mathbf{1 1 - 0 6 - 9 5}$ & $\mathbf{0 7 - 0 9 - 9 7}$ \\
& Draft RA Report to EPAIDHW & $12-02-99$ & $08-01-01$ \\
& Draft O\&M Report to EPAIDHW & $01-04-00$ & $08-24-01$
\end{tabular}

\begin{tabular}{lllll}
\hline OU 7-06 & Final Informal Report & $09-20-95$ & & \\
& & & $08-10-93$ & $09-30-93$ \\
\hline OU 7-08 & Draft RI/FS Report to EPAIDHW & $07-06-93$ & $04-12-94$ & $07-31-94$ \\
& Draft ROD to EPAIDHW & $03-08-94$ & $08-04-94$ & \\
& Final ROD Approved & $06-29-94$ & $09-09-94$ \\
& Draft RD/RA SOW to EPAIDHW & $08-04-94$ & $01-24-95$ & \\
Draft RD Work Plan to EPAIDHW & $12-12-94$ & $05-23-95$ \\
& Remedial Design to EPAIDHW & $04-18-95$ & $06-28-95$
\end{tabular}


Draft O\&M Report to EPAIDHW

05-01-01

06-06-01

Draft RA Report to EPAIDHW

05-22-01

06-27-01

\begin{tabular}{ll}
\hline OU 7-10 & Final ROD Approved \\
& Draft RD/RA SOW to EPAIDHW \\
& DG\&G/DOE-ID Approve LPT Remediation
\end{tabular}

$\begin{array}{ll}03-30-93 & 07-16-93 \\ 04-20-93 & 08-05-93 \\ 06-02-95 & 01-02-97\end{array}$

\begin{tabular}{ll}
\hline OU 7-12 & Draft ROD to EPAIDHW \\
& Final ROD Approved \\
& Draft SOW-RD Work Plan to EPAIDHW \\
& Draft Design to EPAIDHW \\
& Draft RA Work Plan to EPAIDHW \\
& Draft RA Report to EPAIDHW \\
& Draft O\&M Report to EPAIDHW
\end{tabular}

03-31-93

12-23-93

$01-28-94$

$03-31-93$

$05-11-94$

$10-31-93$

07-13-93

08-16-94

10-15-93

08-16-94

10-15-93

03-29-95

05-27-94

03-01-95

\begin{tabular}{ll}
\hline OU 7-13 & Draft SOW to EPAIDHW \\
Draft IA SOW to EPAIDHW \\
Draft RI/FS Work Plan to EPAIDHW \\
Draft IA ROD to EPAIDHW \\
Draft RI/FS Report to EPAIDHW \\
IA ROD Approved \\
Draft SOW-RD Work Plan to EPAIIDHW \\
Draft Design to EPAIDHW \\
Draft RA Work Plan to EPAIDHW \\
Draft RA Report to EPAIDHW \\
Draft O\&M Report to EPAIDHW \\
Draft ROD/Resp. Summary to EPAIDHW \\
Final ROD Approved \\
Draft SOW-RD Work Plan to EPAIDHW \\
Draft Design to EPAIDHW \\
Draft RA Work Plan to EPAIDHW \\
Draft RA Report to EPAVIDHW \\
Draft O\&M Report to EPAIDHW
\end{tabular}

04-29-94

04-29-94

09-30-94

04-25-95

05-28-96

08-17-95

03-22-95

08-07-95

08-07-95

04-03-01

05-02-01

04-01-97

08-01-97

09-23-98

02-09-99

$02-09-99$

11-02-99

12-03-99
11-17-94

06-27-94

04-28-95

06-21-95

08-19-97

11-20-95

10-13-95

01-25-96

04-15-96 06-11-96

04-15-96 06-11-96

12-04-01

01-10-02

02-06-02

03-08-02

06-23-98

10-23-98

02-04-99

06-14-99

06-14-99

03-16-00

04-13-00
08-31-95

$01-31-96$

$09-30-97$

07-31-98

\begin{tabular}{|c|c|c|c|}
\hline $0 \cup 7-14$ & $\begin{array}{l}\text { Draft SOW to EPAIDHW } \\
\text { Draft RI/FS Work Plan to EPAIDHW } \\
\text { Draft RI/FS Report to EPAIDHW } \\
\text { Draft ROD to EPAIDHW } \\
\text { Final ROD Approved }\end{array}$ & $\begin{array}{l}10-27-95 \\
04-01-96 \\
11-25-97 \\
03-16-99 \\
08-09-99\end{array}$ & \\
\hline OU 10-02 & Draft Summary Report to EPAIDHW & $12-05-97$ & \\
\hline OU 10-03 & $\begin{array}{l}\text { Draft Summary Report to EPAIDHW } \\
\text { Draft SOW to EPAIDHW } \\
\text { Draft ROD to EPAIDHW } \\
\text { Final ROD Approved } \\
\text { Draft SOW to EPAIDHW } \\
\text { Draft Design to EPAIDHW } \\
\text { Draft RA Work Plan to EPAIDHW } \\
\text { Draft RA Report to EPAIDHW }\end{array}$ & $\begin{array}{l}12-19-96 \\
05-19-97 \\
06-11-98 \\
11-19-98 \\
10-22-98 \\
03-15-99 \\
03-15-99 \\
05-03-01\end{array}$ & $\begin{array}{l}03-03-99 \\
07-13-99 \\
07-13-99 \\
08-31-01\end{array}$ \\
\hline
\end{tabular}

07-31-96

12-31-96

07-22-98

08-31-98

10-29-99

06-30-99

04-04-00 
Draft O\&M Report to EPAIDHW

03-09-01 07-09-01

\begin{tabular}{ll}
\hline OU 10-04 & Draft SOW to EPAIDHW \\
& Draft RI/FS Work Plan to EPAIDHW
\end{tabular}

Draft RI/FS Report to EPAIDHW

02-19-98

06-23-98

Draft ROD to EPAIDHW

02-07-00

12-07-00

Final ROD Approved

04-18-01

07-25-00

06-30-98

$11-30-98$

07-31-00

05-31-01

OU $10-05$

Draft RA Report to EPAIIDHW

03-10-94 

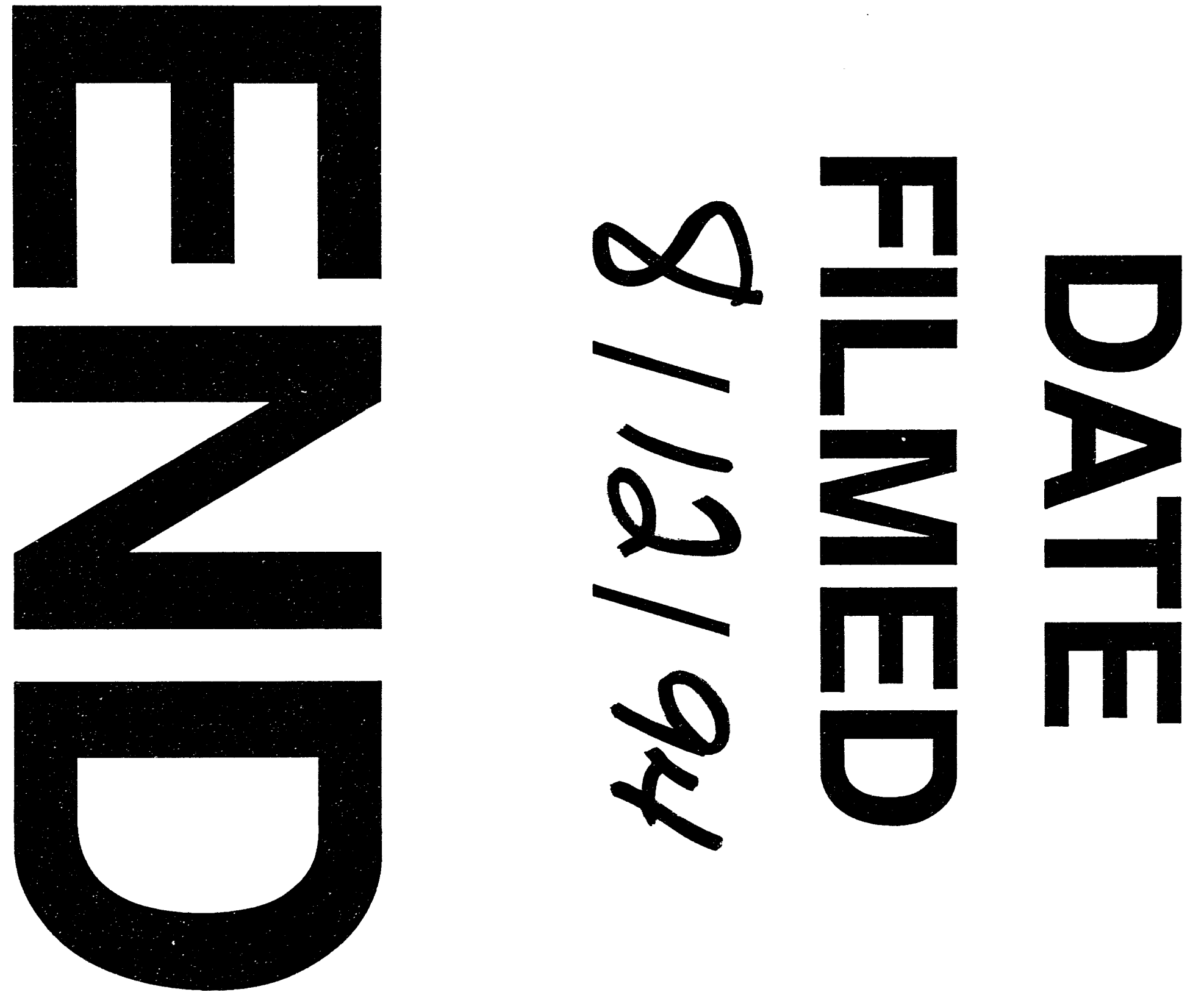

$\underset{D}{0}$
$\stackrel{0}{0}$
$H$ 
